\title{
PSEUDOCONVEX CLASSES OF FUNCTIONS. III CHARACTERIZATION OF DUAL PSEUDOCONVEX CLASSES ON COMPLEX HOMOGENEOUS SPACES
}

\author{
ZBIGNIEW SLODKOWSKI
}

\begin{abstract}
Invariant classes of functions on complex homogeneous spaces, with properties similar to those of the class of plurisubharmonic functions, are studied. The main tool is a regularization method for these classes, and the main theorem characterizes dual classes of functions (where duality is defined in terms of the local maximum property). These results are crucial in proving a duality theorem for complex interpolation of normed spaces, which is given elsewhere.
\end{abstract}

1. Introduction. Pseudoconvex classes of functions, which generalize the wellknown classes of convex, subharmonic, plurisubharmonic and $q$-plurisubharmonic functions, were introduced and studied in the abstract context in [11], and in [12] a complete description of translation invariant pseudoconvex classes on $R^{N}$ was given.

Pseudoconvex classes were applied in [13] to complex interpolation of normed or quasinormed spaces parametrized by points of a subset of a several-dimensional complex homogeneous space (in the spirit of Coifman et al. $[\mathbf{3}, \mathbf{4}]$ ). The exposition in [13], and especially the duality theorem for complex interpolation families of normed spaces proved therein, relied on a characterization of the class of functions which is dual, in a certain sense, to a given one. The main aim of this paper is to prove this characterization (Theorem 4.9 below). Alongside, we generalize to the context of complex homogeneous spaces some of the results of [12].

For basic definitions, notation and background the reader is referred to [11, §1]. We recall, however, the axioms. A class of functions on a topological space $M$ is called generalized pseudoconvex if $M$ is locally compact and axioms (1.1)-(1.6) hold. Below $U$ and $V$ denote open subsets of $M$.

$$
\begin{aligned}
& P(U) \subset \operatorname{usc}(U) . \\
& \text { If } V \subset U \text { and } u \in P(U) \text {, then } u \mid V \in P(V) . \\
& \text { If } u_{n} \in P(U), u_{n}(x) \searrow u(x), x \in U \text {, then } u \in P(U) . \\
& \text { If }\left(u_{t}\right)_{t \in T} \subset P(U) \text { and } u(x)=\sup _{t \in T} u_{t}(x) \text { is locally bounded from } \\
& \text { the above, then } u^{*} \in P(U) .
\end{aligned}
$$

Received by the editors June 1, 1987.

1980 Mathematics Subject Classification (1985 Revision). Primary 32F05; Secondary 32M10, $31 \mathrm{D} 05$.

Key words and phrases. Pseudoconvex classes, subharmonic functions, $q$-plurisubharmonic functions, dual classes of functions, complex homogeneous spaces. 

If $u \in P(U)$ and $C \in R$, then $(u+C) \in P(U)$.

In this paper, $M$ will be a complex homogeneous space $M=K / H$, where $K$ is a real Lie group, $H$ its closed subgroup, and the maps $t_{g}: M \rightarrow M$, where $t_{g}\left(g_{1} H\right)=\left(g g_{1}\right) H$, are biholomorphic with respect to the given complex structure of $M$. In $\S 2$, we approximate functions of an invariant (in the sense of (1.9) below, with $n=0$ ), generalized pseudoconvex class $P$ by functions of the same class whose second-order derivatives are measures (cf. Corollary 2.9).

Applications to complex interpolation of normed spaces in [13] require studying generalized pseudoconvex classes $P$ on the trivial bundle $\widetilde{M}=M \times \mathrm{C}^{n}$. (Note that $\widetilde{M}$ is also a complex homogeneous space since $\widetilde{M}=\widetilde{K} / \widetilde{H}$, where $\widetilde{K}=K \oplus \mathbf{C}^{n}$, $\widetilde{H}=H \oplus\{0\}$.) In $\S \S 4$ and 5, we will assume that $P$ satisfies also conditions (1.7)(1.9) below (and is, consequently, pseudoconvex; cf. [11, Definition 1.4, Proposition 1.7]).

(1.8)(i) In case it is defined on $M$, class $P$ is complex in the sense that, if $u \in P$ and $v$ is plurisubharmonic, then $(u+v) \in P$.

(1.8)(ii) In case it is defined on $\widetilde{M}=M \times \mathbf{C}^{n}$, class $P$ is admissible in the following sense. If $U \subset \widetilde{M}, u \in P(U)$, and $v(z, w)$ is a function plurisubharmonic on $U$ in $(z, w)$ and such that slice functions $w \rightarrow$ $v(z, w)$ are locally convex, then $(u+v) \in P$.

$$
\begin{aligned}
& \text { If } u \in P(U), U \subset \widetilde{M}=M \times \mathbf{C}^{n}, M=K / H, \text { then } u \circ t_{g, w} \in \\
& P\left(t_{g, w}^{-1}(U)\right) \text {, where } t_{g, w}\left(g_{1} H \oplus w_{1}\right)=\left(g g_{1}\right) H \oplus\left(w+w_{1}\right), g, g_{1} \in K, \\
& w, w_{1} \in \mathbf{C}^{n} \text {. }
\end{aligned}
$$

In $\S 3$, we assign to an admissible class of functions on $\widetilde{M}$ the set of forms on $T_{p}(\widetilde{M})$ which are sums of the complex Hessian in $(z, w)$ and of the real Hessian in $w$, for $u \in P$. Properties of this object to a large extent determine properties of $P$, and are crucial in proving the main duality result, Theorem 4.9. The latter, as well as other facts of $\S 4$, are proved for special pseudoconvex classes $P$ on $\widetilde{M}$ which are invariant with respect to biholomorphic bundle automorphisms $(z, w) \rightarrow\left(z, T_{z}(w)\right)$, where $z \in M, T_{z}: \mathbf{C}^{n} \rightarrow \mathbf{C}^{n}$ is a $\mathbf{C}$-affine map. This condition is a natural one, as far as complex interpolation families of normed spaces are concerned. (In [13] we define interpolating norms in terms of such class $P$.)

The results of this paper can be substantially generalized; some indications in this direction are given in $\S 5$.

2. Approximation by functions with lower bounded Hessian on differentiable manifolds. We first define the class of functions with lower bounded Hessian, denoted further by $C_{-\infty, 10 c}^{1,1}$, on a differentiable manifold $M$. (The previous definition, for the Euclidean case $M=R^{N}$ (cf. $\left.[9, \S 2]\right)$ was formulated in terms of convex functions, and so is not immediately applicable in the general context.) We will then show that $P \cap C_{-\infty, 10 c}^{1,1}$ is dense in $P$, whenever $P$ is a generalized pseudoconvex class on $M$ satisfying a suitable invariance condition (cf. Corollary 2.9). 
PROPOSITION 2.1 (FOLK). Let $K \subset U \subset R^{N}$, where $K$ is compact and $U$ open. Let $r=\min \operatorname{dist}(K, \partial U)$. Then, for every $M>0$, there is $C>0$ (e.g. $C=2 M / r)$ such that, for every locally convex function $v$ on $U$ with $\sup _{U}|v| \leq M$, its restriction to $K$ is Lipschitz (of order 1) with Lipschitz constant not exceeding C.

As in $[9, \S 2]$, we denote that $u \in C_{L}^{1}(U)$, where $L \geq 0$ and $U \subset R^{N}$, if the function $x \rightarrow u(x)+\frac{1}{2} L|x|^{2}$ is locally convex. Below the norm of a symmetric or Hermitian matrix is maximum modulus of its eigenvalues.

LEMMA 2.2. If $u: U \rightarrow R$ is a locally convex function and $F=\left(f_{1}, \ldots, f_{N}\right): V$ $\rightarrow U$ is a $C^{(2)}$-smooth map, where $U \subset R^{N}, V \subset R^{N^{\prime}}$ are open sets, then for every relatively compact subset $H$ of $V$, the function $v=u \circ F$ can be represented (on $H$ ) as the difference of a locally convex and of a smooth function. Precisely, $v \mid H \in C_{L}^{1}(H)$, where $L=(2 / r)\|u\|_{U}\left\|d_{2} F\right\|_{H}, r=\min \operatorname{dist}(F(\bar{H}), \partial U),\|u\|_{U}=\sup _{y \in U}|u(y)|$ and $\left\|d_{2} F\right\|_{H}=\sum_{j=1}^{N} \sup _{x \in H}\left\|\left(\operatorname{Hess} f_{j}\right)(x)\right\|$.

Proof. Assume first that $u$ is smooth. By the chain rule,

$$
\frac{\partial^{2} v(x)}{\partial x_{j} \partial x_{k}}=\sum_{r, s=1}^{N} \frac{\partial^{2} u}{\partial y_{r} \partial y_{s}} \frac{\partial f_{r}}{\partial x_{j}} \frac{\partial f_{s}}{\partial x_{k}}+\sum_{r=1}^{N} \frac{\partial u}{\partial y_{r}} \frac{\partial^{2} f_{r}}{\partial x_{j} \partial x_{k}},
$$

$j, k=1,2, \ldots, N^{\prime}$. By the local convexity of $u(y)$, the part of Hess $v(x)$ corresn :ing to the terms of the first sum is a positive semidefinite form, say $A_{1}$, sucli tnat $($ Hess $v)(x)=A_{1}+A_{2}$, where

$$
A_{2}=\sum_{r=1}^{N}\left(\frac{\partial u}{\partial y_{r}}\right)(F(x))\left(\operatorname{Hess} f_{r}\right)(x)
$$

By Proposition 2.1, if $x \in H$, then

$$
\left\|A_{2}\right\| \leq\|\operatorname{grad} u(y)\| \cdot\left\|\left(d_{2} F\right)(x)\right\| \leq L .
$$

Thus, all the eigenvalues of (Hess $v)(x), x \in H$, are greater than or equal to $-L$, and so $v \mid H \in C_{L}^{1}(H)$; cf. [9, Propositions 2.2(iii), 2.3(ix)].

If $u$ is an arbitrary, locally convex function, fix $\varepsilon>0$ and choose a descending sequence of smooth convex functions $\left(u_{n}\right)$, defined in $U_{\varepsilon}=\{x \in U: \operatorname{dist}(x, \partial U)>$ $\varepsilon\}$ and such that $u_{n}(x) \rightarrow u(x), x \in U_{\varepsilon}$, and $\left\|u_{n}\right\|_{U_{\varepsilon}} \leq\|u\|_{U}+\varepsilon$. Let $v_{n}=u_{n} \circ F$. Then $v_{n} \mid H \in C_{L(\varepsilon)}^{1}(H)$, where $L(\varepsilon)=(2 /(r-\varepsilon))(\|u\|+\varepsilon)\left\|d_{2} F\right\|_{H}$.

Since $v_{n}(x) \searrow v(x), x \in F^{-1}\left(U_{\varepsilon}\right), v \mid H \in C_{L(\varepsilon)}^{1}(H)$, by [9, Proposition 2.3(vii)]. Since $\lim _{\varepsilon \rightarrow 0} L(\varepsilon)=L, v \mid H \in C_{L}^{1}(H)$; cf. [9, Proposition 2.3]. Q.E.D.

The last lemma motivates the following

DEFINITION 2.3. Let $M$ be a differentiable $C^{(2)}$-manifold. A function $u: U \rightarrow R$ ( $U$ open) is said to have locally lower bounded Hessian (i.e. $u \in C_{-\infty, 10 c}^{1,1}(U)$ ) if, for every $x \in U$, there is a coordinate neighborhood $U_{x}$ of $x$, such that $u \mid U_{x} \cap U=$ $u_{1}-u_{2}$, where $u_{2}$ is smooth and $u_{1}$ is convex with respect to some coordinate system in $U_{x}$.

REMARK 2.4. If, in the context of the last definition, we fix a family of coordinate systems $\left(U_{t}, F^{t}\right), t \in T$, where $\bigcup U_{t}=U$, and an open covering $\left\{H_{t}\right\}_{t \in T}$ of $U$ such that every $H_{t}$ is relatively compact in $U_{t}, t \in T$, then $u: U \rightarrow R$ is of class 
$C_{-\infty, \text { loc }}^{1,1}(U)$, if and only if for each $t \in T, u \mid H_{t} \in C_{L(t)}^{1}\left(H_{t}\right)$, with some constant $L(t) \geq 0$, with respect to the coordinate system $F^{t}=\left(f_{1}^{t}, \ldots, f_{N}^{t}\right)$.

The remark and the next corollary are direct consequences of Lemma 2.2.

Corollary 2.5. If $V, U$ are $C^{(2)}$-manifolds, $u \in C_{-\infty, \text { loc }}^{1,1}(U)$, and $\Phi: V \rightarrow U$ is a $C^{(2)}$-smooth mapping, then $u \circ \Phi \in C_{-\infty, \text { loc }}^{1,1}(V)$.

We will now generalize results of $[\mathbf{1 2}, \S 2]$; cf. also [9, Theorem 2.9] to the setting when the group of translation of $R^{N}$ is replaced by a local Lie group $W$ of diffeomorphisms.

Let $W$ be a real local Lie group, with unit element $e$, product $g_{1} g_{2} \in W$ defined for $g_{1}, g_{2} \in W_{1}$, where $W_{1}$ is a neighborhood of $e$, and inverse $g^{-1} \in W$ for $g \in W$. Assume that an open subset $M_{0} \subset M$ and maps $t_{g}: M_{0} \rightarrow M, g \in W$, satisfying conditions (2.1)-(2.5) are given.

$t_{g}$ is a diffeomorphism onto its range, for every $g \in W$.

The map $(x, g) \rightarrow t_{g} x: M_{0} \times W \rightarrow M$ is $C^{(2)}$-differentiable.

For every $x \in M_{0}$, the map $g \rightarrow t_{g} x: W \rightarrow M$ has differential of constant rank equal to $\operatorname{dim} M$.

$t_{e} x=x, \quad x \in M_{0}$.

For every compact $K \subset M_{0}$, there is a neighborhood $W^{*}$ of $e$, $W^{*} \subset W$, such that whenever $g, h \in W^{*}$, then $t_{g} \circ t_{h}$ is defined near $K$ and $t_{g} \circ t_{h}=t_{g h}$.

In the following lemma we generalize the operation of supremum-convolution introduced in $[\mathbf{9}, \S 2]$; cf. also $[\mathbf{1 2}, \S 2]$.

LEMMA 2.6. Let $M$ be a differentiable manifold of class $C^{(2)}$, and let $M_{0}, W$, $\left\{t_{g}\right\}_{g \in W}$ satisfy conditions (2.1)-(2.5). Let $H$ be an open, relatively compact set with $\bar{H} \subset M_{0}$. Then there is a neighborhood $W_{0}$, with $e \in W_{0} \subset W$, such that for every $u \in \operatorname{usc}\left(M_{0}\right) \cap L^{\infty}$ and for every function $\rho \in C^{(2)}(W, R)$ satisfying conditions

$$
\begin{gathered}
\rho(e)=0, \\
\rho \mid W \backslash W_{0} \leq-2\|u\|_{\infty},
\end{gathered}
$$

the function

$$
\rho *_{s} u(x):=\sup _{g}\left(\rho(g)+u\left(t_{g} x\right)\right)
$$

is of class $C_{-\infty, 10 c}^{1,1}$ on $H$.

PROOF. Fix $p \in H$; it is enough to show that $\rho *_{s} u$ is of $C_{-\infty, \text { loc }}^{1,1}$ class in a neighborhood of $p$. By (2.3) and the constant rank theorem [8, Theorem 1.3.14], there is a neighborhood $W_{0}$ of $e$, a coordinate neighborhood $H_{p}$ of $p$, and a $C^{(2)}$ smooth map $s: \operatorname{nbhd}\left(\bar{H}_{p}\right) \rightarrow W$, such that

$$
s(p)=e ; \quad t_{s(x)} p=x, \quad x \in \operatorname{nbhd}\left(\bar{H}_{p}\right),
$$


$s\left(\bar{H}_{p}\right) \subset W_{0}$, where $g=g_{1} g_{2} g_{3}^{-1}$ is defined and $t_{g} x \in M_{0}$, whenever $g_{1}, g_{2}, g_{3} \in \bar{W}_{0}$ and $x \in \bar{H}$.

ASSERTION. There is a $C^{(2)}$-map $\left(x_{0}, g_{0}, x\right) \rightarrow \mu\left(x_{0}, g_{0}, x\right)$ of a neighborhood of $\bar{H}_{p} \times \bar{W}_{0} \times \bar{H}_{p}$ into $W$, such that

$$
\begin{aligned}
\mu\left(x_{0}, g_{0}, x_{0}\right)= & g_{0}, \quad\left(x_{0}, g_{0}\right) \in \operatorname{nbhd}\left(\bar{H}_{p} \times \bar{W}_{0}\right), \\
& t_{\mu\left(x_{0}, g_{0}, x\right)} x=t_{g_{0}} x_{0} .
\end{aligned}
$$

Let $\mu\left(x_{0}, g_{0}, x\right)=g_{0} s\left(x_{0}\right) s(x)^{-1}$. Then (2.11) and smoothness are obvious. As for (2.12), $t_{\mu} x=t_{g_{0}} t_{s\left(x_{0}\right)} t_{s(x)}^{-1} x=t_{g_{0}} t_{s\left(x_{0}\right)} p=t_{g_{0}} x_{0}$, by (2.9). ( $W_{0}$ has to be small enough so that (2.5) holds.)

Actually, the assertion could be proven using only conditions (2.1)-(2.4) without any local group structure on $W$. In the remainder of the proof only (2.4) and the assertion are used.

Denote $v(x)=\sup \left\{\rho\left(\mu\left(x_{0}, g_{0}, x\right)\right)+u\left(t_{g_{0}} x_{0}\right): g_{0} \in \bar{W}_{0}, x_{0} \in \bar{H}_{p}\right\}$, for $x \in$ $\operatorname{nbhd}\left(\bar{H}_{p}\right)$. Observe that $v(x)=\rho *_{s} u(x)$ for $x \in \operatorname{nbhd}\left(\bar{H}_{p}\right)$, assuming (2.6) and (2.7). On one hand, if we let $g=\mu\left(x_{0}, g_{0}, x\right)$, then $g \in W$ and $t_{g} x \in M_{0}$ by (2.10), and $t_{g} x=t_{g_{0}} x_{0}$ by $(2.12)$. Hence, $\rho\left(\mu\left(x_{0}, g_{0}, x\right)\right)+u\left(t_{g_{0}} x_{0}\right)=\rho(g)+u\left(t_{g} x\right)$, and so $v(x) \leq \rho *_{s} u(x)$ for $x \in \bar{H}_{p}$. Concerning the opposite inequality, note first that

$$
\rho *_{s} u(x)=\sup _{g \in \bar{W}_{0}}\left(\rho(g)+u\left(t_{g} x\right)\right) .
$$

Indeed, by (2.7) and (2.6), if $g \in W \backslash W_{0}$, then $\rho(g)+u\left(t_{g} x\right) \leq-2\|u\|_{\infty}+\|u\|_{\infty} \leq$ $0-\|u\|_{\infty} \leq \rho(e)+u\left(t_{e} x\right)$. If $g \in \bar{W}_{0}, x \in \bar{H}_{p}$, then, by $(2.11), \rho(g)+u\left(t_{g} x\right)=$ $\rho(\mu(x, g, x))+u\left(t_{g} x\right) \leq v(x)$, and so, by $(2.13), \rho *_{s} u(x) \leq v(x)$ near $\bar{H}_{p}$. By the previous inequality, $\rho *_{s} u$ and $v$ are equal near $\bar{H}_{p}$. We will conclude the proof by showing that $v \in C_{-\infty, \text { loc }}^{1,1}$

By the assertion, the function $\left(x_{0}, g_{0}, x\right) \rightarrow \rho\left(\mu\left(x_{0}, g_{0}, x\right)\right)$ is $C^{(2)}$ near $\bar{H}_{p} \times$ $\bar{W}_{0} \times \bar{H}_{p}$. Fix a coordinate system on a neighborhood of $\bar{H}_{p}$. Clearly, for some neighborhood $H^{*}$ of $\bar{H}_{p}$, all the functions

$$
x \rightarrow \rho\left(\mu\left(x_{0}, g_{0}, x\right)\right): H^{*} \rightarrow R, \quad\left(x_{0}, g_{0}\right) \in \bar{H}_{p} \times \bar{W}_{0},
$$

have uniformly bounded Hessian, say by common constant $L>0$, and the same holds for the family of functions

$$
x \rightarrow \rho\left(\mu\left(x_{0}, g_{0}, x\right)\right)+u\left(t_{g_{0}} x_{0}\right), \quad x_{0} \in \bar{H}_{p}, g_{0} \in \bar{W} .
$$

By [9, Proposition 2.3(ix)], all these functions belong to the class $C_{L}^{1}\left(H^{*}\right)$ (relative to the fixed coordinate system). Since $\|u\|_{\infty}<\infty$, the family (2.14) is uniformly bounded and so its supremum, namely $v$, is of class $C_{L}^{1}\left(H^{*}\right)$ by [9, Proposition 2.3(iv)]. By Remark 2.4, the proof is complete. Q.E.D.

COROLLARY 2.7. Let $M, M_{0}, W,\left\{t_{g}\right\}_{g \in W}$ be as in Lemma 2.6. (Local group structure on $W$ and (2.1)-(2.3), (2.5) are not assumed; continuity of $(x, g) \rightarrow$ $t_{g} x: M_{0} \times W \rightarrow M$ and (2.4) are assumed instead.) Let $P$ be a generalized pseudoconvex class of functions on $M$, such that

$$
u \circ t_{g} \rightarrow P\left(t_{g}^{-1}(U)\right), \quad \text { whenever } g \in W, u \in P(U), t_{g}^{-1}(U) \subset M_{0} .
$$


If $\bar{H} \subset M_{0}, \bar{H}$ compact and $W_{0}$ is a neighborhood of $e$, such that $t_{g}(\bar{H}) \subset M_{0}$ for $g \in W_{0}$, then, for every $u \in P\left(M_{0}\right)$ and upper-bounded function $\rho: W \rightarrow R$, satisfying conditions (2.6), (2.7), the usc regularization of $\rho *_{s} u$ is of class $P$ on $H$.

ProOF. By (2.13), $\rho *_{s} u \mid \bar{H}$ is the supremum of functions $\rho(g)+u \circ t_{g}, g \in$ $\bar{W}_{0}$, which are of class $P$ by (2.15) and (1.5). Since these functions are uniformly bounded from above, the usc regularization of $\rho *_{s} u \in P(H)$, by axiom (1.4). Q.E.D.

LEMMA 2.8. Let $M$ be a $C^{(2)}$-manifold, $W$ a local Lie group, $t_{g}: M_{0} \rightarrow M, g \in$ $W$, where $M_{0} \subset M$. Assume, (2.1)-(2.5) hold. Let $P$ be a generalized pseudoconvex class of functions on $M$ (i.e. (1.1)-(1.6) hold) satisfying (2.15). Then, for every $u \in P(U), U \subset M_{0}$, and for every compact set $K \subset U$, there is a sequence $\left(u_{k}\right)_{k=1}^{\infty}$ of functions on $M_{0}$, such that $u_{k} \mid K \in P(\operatorname{nbhd} K) \cap C_{-\infty, \text { loc }}^{1,1}(\operatorname{nbhd} K)$ and $u_{k}(x) \searrow$ $u(x), x \in K$.

ProOF (SKeTCH). Denote by $B^{\infty}\left(M_{0}\right)$ the space of bounded Borel measurable functions on $M_{0}$. We construct first (nonlinear) operators $R_{n}: B^{\infty}\left(M_{0}\right) \rightarrow$ $B^{\infty}\left(M_{0}\right)$ with the following properties:

$$
\left(R_{n} u\right)(x) \geq\left(R_{n+1} u\right)(x) \geq u(x), \quad x \in M_{0} ;
$$

$$
\begin{aligned}
& \lim _{n}\left(R_{n} u\right)(x)=u(x) \text { for every } x \text { at which function } u \text { is upper } \\
& \text { semicontinuous; }
\end{aligned}
$$

whenever $U \subset M_{0}, u \in B^{\infty}\left(M_{0}\right) \cap P(U), \bar{H}$ is compact and $\bar{H} \subset U$, then there is $n_{0}$, such that $\left(R_{n} u\right) \mid H \in P(H) \cap C_{-\infty, \text { loc }}^{\mathbf{1 , 1}}(H)$, for $n \geq n_{0}=n_{0}\left(H,\|u\|_{\infty}\right)$.

To define $R_{n}$, choose a basis $\left(W_{n}\right)$ of neighborhoods of $e$, such that $\bar{W}_{n+1} \subset W_{n}$, $n=1,2, \ldots$, a sequence $\left(C_{n}\right) \subset R$, with $C_{n} \nearrow \infty$, and a sequence of smooth functions $\rho_{n}: W \rightarrow R, n=1,2, \ldots$, such that

$$
\begin{gathered}
\rho_{n}(0)=0 \geq \rho_{n}(g), \quad g \in W, n=1,2, \ldots, \\
\rho_{n}(g) \geq \rho_{n+1}(g), \quad g \in W, n=1,2, \ldots, \\
\rho_{n}(g)=-C_{n}, \quad g \in W \backslash \bar{W}_{n} .
\end{gathered}
$$

Let $R_{n} u(x)=\rho_{n} *_{s} u(x)=\sup \left\{\rho_{n}(g)+\tilde{u}\left(t_{g} x\right): g \in W\right\}, x \in M_{0}$, where $\tilde{u}(x)=0$, if $x \in M_{0} \backslash$ domain $(u)$.

Clearly, (2.16) holds by (2.19) and (2.20). By (2.13), it holds

$$
\left(R_{n} u\right)(x)=\sup \left\{\rho_{n}(g)+u\left(t_{g} x\right): g \in \bar{W}_{n}\right\},
$$

provided $2\|u\|_{\infty} \leq C_{n}$. This and (2.16) implies (2.17). Finally, Lemma 2.6, Corollary 2.7 and (2.22) imply (2.18).

Shrinking $U$, if necessary, we can assume that $U$ is relatively compact and $u$ bounded from the above on $U$. By axiom (1.6), $P(U)$ contains a bounded function, say $h$. By (1.4), (1.5), the function $x \rightarrow 2^{-k}+\max (u(x),-k+h(x))$ is of class $P$ on $U$. It is now easy to check, using (2.16)-(2.18), that, given $u \in P(U)$ and a compact set $K \subset U$, we can choose a sequence $n_{k} \nearrow \infty$, such that the functions $u_{k}(x)=R_{n_{k}}\left(2^{-k}+\max (u(x),-k+h(x))\right)$ have all the required properties. We omit further details (similar to those in [12, $\S 2$ or 9, Proof of Theorem 2.9]). Q.E.D. 
COROLLARY 2.9. Let $M$ be a real homogeneous space $K / H$ and $P$ be a generalized pseudoconvex class on $M$ (i.e. (1.1)-(1.6) hold), invariant in the sense of (1.9) (with $n=0$ ). Then $P \cap C_{-\infty, 10 c}^{1,1}$ is dense in $P$ in the following sense: whenever $u \in P(U), K \subset U \subset M, K$ is compact, then there exist functions $u_{n} \in P(\operatorname{nbhd} K) \cap C_{-\infty, \text { loc }}^{1,1}(\operatorname{nbhd} K), n=1,2, \ldots$, such that $u_{n}(x) \searrow u(x), x \in K$.

PROOF. The corollary follows directly from the last lemma and well-known properties of homogeneous spaces; cf. Kobayashi and Nomizu [6, Vol. II, Chapter IX]. (Conditions (2.1)-(2.5) hold with $W=K, M_{0}=M=K / H$.) Q.E.D.

3. Invariant classes of functions on complex homogeneous spaces. From now on, we consider generalized pseudoconvex classes $P$ on $\widetilde{M}=M \times \mathbf{C}^{n}$, where $M=K / H$ is a complex homogeneous space, related to the complex structure of $\widetilde{M}$ via the admissibility condition (1.8)(ii). The approximation result of $\S 2$ (based on axiom (1.9)) enables us to study the relation between class $P$ and Hessians of functions in $P$ (similarly as it was done in $[\mathbf{1 2}, \S 3]$ for the case $M=R^{N}$ ). In this section we discuss only those facts which will be needed in the proof of Theorem 4.9 .

Fix $p=\left(z_{0}, w_{0}\right) \in \widetilde{M}=M \times \mathbf{C}^{n}$. (At this stage $M$ is just a complex manifold $M$ with $\operatorname{dim}_{C} M=k$.) Let $P$ be a class of functions on $\widetilde{M}$, satisfying (1.8)(ii). We will assign to $P$ a class $Y_{p}$ of homogeneous quadratic forms on $T_{p}(\widetilde{M})$ (= the tangent space at $p$ ).

We will call a system of coordinates on $\widetilde{M}$ admissible, if it is of the form $\left(\varsigma_{1}(z), \ldots, \varsigma_{k}(z), w_{1}, \ldots, w_{n}\right)$, where $\left(\varsigma_{1}, \ldots, \zeta_{k}\right)$ form a holomorphic coordinate system on $M$ and $w_{1}, \ldots, w_{n}$ are the standard coordinates on $\mathbf{C}^{n}$. To develop the definition of $Y_{p}$, fix an admissible coordinate system $\left(\varsigma_{1}, \ldots, \varsigma_{k}, w_{1}, \ldots, w_{n}\right)$ at $p$ and consider any function $u$ of class $P \cap C_{-\infty, \text { loc }}^{1,1}$ near $p$, which has the secondorder Peano differential at $p$. (There are plenty of such functions, by Corollary 2.9, if (1.9) is assumed; then the second-order Peano differential exists a.e. by $[\mathbf{1}, \mathbf{2}]$; cf. also comments in $\left[\mathbf{9}, \S 3\right.$, p. 311].) Let $X=\left(Z_{1}, \ldots, Z_{k}, W_{1}, \ldots, W_{n}\right)$ denote (complex) coordinates of a vector in $T_{p}(\widetilde{M})$, with respect to the basis $\partial / \partial \varsigma_{1}, \ldots, \partial / \partial \zeta_{k}, \partial / \partial w_{1}, \ldots, \partial / \partial w_{n}$ corresponding to fixed admissible coordinate system. We split second-order differential of $u$ at $p$ into part (I) and part (II), where

$$
\begin{aligned}
\operatorname{part}(\mathrm{I})= & 2 \operatorname{Re}\left[\sum_{i=1}^{k} \frac{\partial u(p)}{\partial z_{i}} Z_{i}+\sum_{j=1}^{n} \frac{\partial u}{\partial w_{j}} W_{j}\right] \\
& +\operatorname{Re}\left[\sum_{i j} \frac{\partial^{2} u(p)}{\partial z_{i} \partial z_{j}} Z_{i} Z_{j}+\sum_{i q} \frac{\partial^{2} u(p)}{\partial z_{i} \partial w_{q}} Z_{i} W_{q}+\sum_{p j} \frac{\partial^{2} u}{\partial w_{p} \partial z_{j}} Z_{p} W_{j}\right]
\end{aligned}
$$

$$
\operatorname{part}(\mathrm{II})=\operatorname{Re}\left(\sum_{p q} \frac{\partial^{2} u(p)}{\partial w_{p} \partial w_{q}} W_{p} W_{q}\right)+\operatorname{Hess}^{\mathbf{C}} u(p)(X, X)
$$




$$
\begin{aligned}
\operatorname{Hess}^{\mathbf{C}} u(p)(X, X)= & \sum_{i j} \frac{\partial^{2} u}{\partial z_{i} \partial \bar{z}_{j}} Z_{i} \bar{Z}_{j}+\sum_{i q} \frac{\partial^{2} u}{\partial z_{i} \partial \bar{w}_{j}} Z_{i} \bar{W}_{j} \\
& +\sum_{p j} \frac{\partial^{2} u}{\partial \bar{z}_{p} \partial w_{j}} \bar{Z}_{p} W_{j}+\sum_{p q} \frac{\partial^{2} u}{\partial w_{p} \partial \bar{w}_{q}} W_{p} \bar{W}_{q} .
\end{aligned}
$$

Observe now that, if a function $v(z, w)$ satisfies the next condition

$$
\begin{aligned}
& v(z, w) \text { is pluriharmonic in }(z, w) \in M \times \mathbf{C}^{n} \text { and, for every } z \text {, the } \\
& \text { slice function } w \rightarrow v(z, w) \text { is } R \text {-affine, }
\end{aligned}
$$

then its (quadratic) differential consists only of part (I) and this property is true for every admissible coordinate system. Observe now that, given any type I differential at $p$ (in a fixed admissible coordinate system), we can represent it as the full quadratic differential (at $p$ ) of a function $v(z, w)$ satisfying (3.4). Just take $v(z, w)=$ the real part of a suitable quadratic polynomial in $\zeta_{1}(z), \ldots, \zeta_{k}(z), w_{1}, \ldots, w_{n}$ (cf. $(3.1))$. (This polynomial does not include terms $w_{p} w_{q}$.) By adding such $v(z, w)$ to $u(z, w)$, we can freely control part (I) of the differential of $(u+v)$, while part (II) stays unchanged. Since for every such $v$, it holds: $v+P=(-v)+P=P$ (by axiom (1.8)(ii)), we conclude that it is part (II) of a differential which determines whether this differential is equal to the differential of a function in $P \cap C_{-\infty, \text { loc }}^{1,1}$.

So far, the admissible coordinate system $\zeta_{1}, \ldots, \zeta_{k}, w_{1}, \ldots, w_{n}$ was kept fixed. Since the change from one to another admissible coordinate system is of the form $\varsigma_{1}^{\prime}=\varsigma_{1}^{\prime}(\varsigma), \ldots, \zeta_{k}^{\prime}=\zeta_{k}^{\prime}(\varsigma), w_{1}^{\prime}=w_{1}, \ldots, w_{n}^{\prime}=w_{n}$, with $\varsigma \rightarrow \zeta^{\prime}$ biholomorphic, computations like in Krantz [7, p. 105], and (3.3), (3.4) imply that part (II) is functorial and defines, independently on the choice of the admissible coordinate system, a real homogeneous quadratic form on $T_{p}(\widetilde{M})$, which we denote by $\operatorname{Hess}^{\mathrm{II}} u(p)$. The following definition is, by now, justified.

DEFINITION 3.1. (a) We let $h_{p}$ denote the real vector space of real quadratic forms on $T_{p}(\widetilde{M})$ which, relative to some admissible coordinate system, have representation

$$
\begin{aligned}
& A(Z \oplus W)+\operatorname{Re}\langle W, B W\rangle \\
& \quad=\left\langle\bar{Z}, A_{11} Z\right\rangle+\left\langle\bar{Z}, A_{12} W\right\rangle+\left\langle\bar{W}, A_{21} Z\right\rangle+\left\langle\bar{W}, A_{22} W\right\rangle+\operatorname{Re}\langle W, B W\rangle
\end{aligned}
$$

where $\langle\cdot, \cdot\rangle$ denotes the standard $\mathbf{C}$-bilinear product.

(b) We let $h_{p}^{+}$denote the open convex cone in $h_{p}$ consisting of all forms (3.5), such that both the Hermitian part $A(Z \oplus W)$ and the symmetric form $\left\langle\bar{W}, A_{22} W+\right.$ $\operatorname{Re}\langle W, B W\rangle$ are positive definite.

(c) Let $Y_{p}^{\prime}$ be the subset of $h_{p}$ consisting of all forms $\operatorname{Hess}^{\mathrm{II}} u(p)$, where $u \in$ $P \cap C_{-\infty, \text { loc }}^{1,1}$ (near $p$ ) and $u$ has a second-order Peano differential at $p$. We denote by $Y_{p}$ the closure of $Y_{p}^{\prime}$ in $h_{p}$ and call $Y_{p}$ the class of forms representing $P$ at $p$.

REMARK 3.2. By previous discussion, given an admissible coordinate system near $p \in \widetilde{M}$ and a function $u$, with the second-order Peano differential at $p$, there is a function $v$ satisfying (3.4) (in particular, $\operatorname{Hess}^{\mathrm{II}} v(p)=0$ ), such that the secondorder differential at $p$, in a given coordinate system, is equal to $\operatorname{Hess}^{\mathrm{II}} u(p)$.

Proposition 3.3. Let $M$ be a complex manifold, $\widetilde{M}=M \times \mathbf{C}^{n}, p \in \widetilde{M}$, and $P$ be an admissible class of usc functions on $\widetilde{M}$; cf. (1.8)(ii). Then $h_{p}^{+}$is an open 
convex cone, such that $h_{p}^{+} \cap\left(-h_{p}^{+}\right)=\varnothing$, and $h_{p}$, with order determined by $h_{p}^{+}$, is an ordered vector space. The set $Y_{p}^{\prime}$ is an order cone in $h_{p}$ (i.e. if $F \in Y_{p}^{\prime}$ and $A \in h_{p}^{+}$, then $\left.(F+A) \in Y_{p}^{\prime}\right) ; c f .[12$, Definition A.2(i)]. Furthermore, the representing class $Y_{p}$ is a closed order cone and Int $Y_{p} \subset Y_{p}^{\prime}$.

In the further discussion we will use elementary results on order cones in ordered finite-dimensional vector spaces, which were collected in [12, Appendix A].

ProOF OF PROPOSITION 3.3. Properties of $h_{p}^{+}$are obvious.

Let $F \in Y_{p}^{\prime}$ and $F_{+} \in h_{p}^{+}$. Fix an admissible coordinate system $\left(\varsigma_{1}, \ldots, \varsigma_{k}\right.$, $\left.w_{1}, \ldots, w_{n}\right)$ at $p$. Then, there is $u \in P \cap C_{-\infty, \text { loc }}^{1,1}($ nbhd $p)$, such that $F=\operatorname{Hess}^{\text {II }} u(p)$. Let $\varphi$ be a quadratic polynomial in $\varsigma_{1}, \ldots, \zeta_{k}, \bar{\zeta}_{1}, \ldots, \bar{\zeta}_{k}, w_{1}, \ldots, w_{n}, \bar{w}_{1}, \ldots, \bar{w}_{n}$, determined by the representation of $F_{+}$, relative to the admissible coordinate system (cf. (3.5)). Let

$$
v(z, w)=\varphi\left(\varsigma_{1}(z), \ldots, \zeta_{k}(z), \overline{\zeta_{1}(z)}, \ldots, \overline{\zeta_{k}(z)}, w_{1}, \ldots, w_{n}, \bar{w}_{1}, \ldots, \bar{w}_{n}\right) .
$$

Clearly, Hess ${ }^{\mathrm{II}} v(p)=F_{+}$. On the other hand, $v(z, w)$ is plurisubharmonic in $(z, w)$ and convex in $w$, and so, by (1.8)(ii), $(u+v) \in P \cap C_{-\infty, \text { loc }}^{1,1}$. Hence, $F+F_{+}=$ $\operatorname{Hess}(u+v)(p) \in Y_{p}^{\prime}$. Thus, $Y_{p}^{\prime}$ is an order cone.

By [12, Proposition A.5(iii),(v)], both $Y_{p}=\overline{Y_{p}^{\prime}}$ and Int $\left(Y_{p}^{\prime}\right)$ must be order cones, and $Y_{p}^{\prime} \supset \operatorname{Int}\left(Y_{p}\right)$. Q.E.D.

PROPOSITION 3.4. Under assumptions of Proposition 3.3, the class of forms representing $P^{d}$ (= the dual class of functions to $P$; cf. $[11$, Definition 1.11]) at $p \in \widetilde{M}$ is contained in $Y_{p}^{D}=\left\{F \in h_{p}:\left(F+Y_{p}\right) \cap\left(-h_{p}^{+}\right)=\varnothing\right\}$ (= the dual order cone; cf. [12, Definition A.2(iii)]).

ProOF. Consider another order structure on $h_{p}$ determined by the open, convex cone $h^{0}$ consisting of all forms (3.5) which are positive definite. Clearly, $h^{0} \subset h_{p}^{+}$. (If a form (3.5) is positive definite, then its Hermitian part is also positive definite; see e.g. $\left[9\right.$, p. 319].) By [12, Remark A.7], the dual order cones to $Y_{p}$ relative to either structure (i.e. $\left(h_{p}, h_{p}^{+}\right)$and $\left.\left(h_{p}, h^{0}\right)\right)$ are the same. Furthermore, by $[\mathbf{1 2}$, Lemma A.8], $Y_{p}^{D}=\left(\overline{Y_{p}^{\prime}}\right)^{D}=\left(Y_{p}^{\prime}\right)^{D}$ and so $\left(Y_{p}^{\prime}\right)^{D}=\left\{F \in h_{p}:\left(F+Y_{p}^{\prime}\right) \cap\left(-h^{0}\right)=\right.$ $\varnothing\}$. Consequently, if the class of forms representing $P^{d}$ at $p$ is not contained in $Y_{p}^{D}$, then there exist functions $u_{1} \in P(\operatorname{nbhd} p), u_{2} \in P(\operatorname{nbhd} p)$, both of class $C_{-\infty, \text { loc }}^{1,1}$ and having second-order Peano differentials at $p$, such that $\operatorname{Hess}^{\mathrm{II}} u_{1}(p)=$ $F_{1}$, Hess ${ }^{\mathrm{II}} u_{2}(p)=F_{2}$, and $F_{1}+F_{2}$ is negative definite.

Fix an admissible coordinate system $\left(\varsigma_{1}, \ldots, \varsigma_{k}, w_{1}, \ldots, w_{n}\right)$ at $p$. By Remark 3.2 , we can add to $u_{1}+u_{2}$ a function $v(z, w)$ satisfying condition (3.4), so that the second-order Peano differential of $\left(u_{1}+u_{2}+v\right)$ at $p$ is equal to $F_{1}+F_{2}$. Identifying $F_{1}+F_{2}$ with a matrix, we can now write $\left(u_{1}+u_{2}+v\right)(x)=\left(\left(F_{1}+F_{2}\right) K, K\right)+r(K)$, where $K$ is the vector $[\zeta(x)-\zeta(p) ; w(x)-w(p)]$ and $\lim _{x \rightarrow p}\left(r(K) /\|K\|^{2}\right)=0$. Since $\left(\left(F_{1}+F_{2}\right) K, K\right) \leq-\varepsilon\|K\|^{2}$, for some $\varepsilon>0$, function $u_{1}+u_{2}+v$ has strict local maximum at $p$. This contradicts the definition of $P^{d}$; cf. [11, Definition 1.11] because $\left(u_{1}+v\right) \in P$, by (1.8)(ii). Q.E.D.

The separation property introduced in the next definition will serve to localize maximum properties. 
DEFINITION 3.5. We say that a complex manifold $M$ has the separation property, if for every compact set $K \subset M$, the algebra $\operatorname{Hol}(K)$, consisting of all functions holomorphic in a neighborhood of $K$, separates points of $K$.

EXAMPLE. Every open subset of an arbitrary Stein manifold has the separation property in the above sense.

LEMMA 3.6. Let $M$ be a complex manifold with the separation property. Let $K, L$ be compact subsets of $\widetilde{M}=M \times \mathbf{C}^{n}$, such that $L \subset K$, and $u \in \operatorname{usc}(K)$. Assume that $\max u|L<\max u| K$. Then there is a neighborhood of $K$ of the form $V \times \mathbf{C}^{n}$ and a function $v: V \times \mathbf{C}^{n} \rightarrow R$, satisfying condition (3.4) and $x_{0} \in K \backslash L$, such that

$$
(u+v)\left(x_{0}\right)=0>(u+v)(x), \quad x \in K \backslash\left\{x_{0}\right\} .
$$

Proof. Choose a compact neighborhood $X$ of $K$ in $\widetilde{M}$ of the form $X=\bar{V} \times$ $\bar{B}(0, R)$, where $V$ is open in $M, V=\operatorname{Int}(\bar{V})$. Let $A$ be the closed subspace of $C(X)$ consisting of functions of the form

$$
h(z, w)=h_{0}(z)+h_{1}(z) w_{1}+\cdots+h_{n}(z) w_{n},
$$

where $h_{i} \in C(\bar{V}) \cap \operatorname{Hol}(V), i=0,1, \ldots, n$. Applying [11, Lemma A.1 and Proposition A.4] to the function $u_{1} \in \operatorname{usc}(X)$, defined by $u_{1}\left|K=u, u_{1}\right| X \backslash K=-\infty$, we obtain $\rho_{1} \in A$ and $x_{0} \in K \backslash L$, such that

$$
\left(u_{1}+\operatorname{Re} \rho_{1}\right)\left(x_{0}\right)=0>\left(u_{1}+\operatorname{Re} \rho_{1}\right)(x), \quad x \in X \backslash\left\{x_{0}\right\} .
$$

(Note. Functions in $A$ separate points of $X$ by the separation property of $M$, and so assumptions of $\left[\mathbf{1 1}\right.$, Lemma A.1] hold.) Let $\rho(z, w)$ be an extension of $\rho_{1}$ to $V \times \mathbf{C}^{n}$, which is $\mathbf{C}$-affine in $w$. Clearly, $\rho \in \operatorname{Hol}\left(V \times \mathbf{C}^{n}\right)$ and the function $v(z, w)=\operatorname{Re} \rho(z, w)$ satisfies all the requirements of the lemma. Q.E.D.

LEMMA 3.7. Let $M$ be a complex manifold with the separation property (cf. Definition 3.5). Let $\widetilde{M} \cup B$, where $\widetilde{M}=M \times \mathrm{C}^{n}$, be a locally compact topological space, such that $\widetilde{M} \cap B=\varnothing$ and $B$ is closed in $\widetilde{M} \cup B$. Let $K$ be a compact subset of $\widetilde{M} \cup B$ and $u \in \operatorname{usc}(K)$. Assume that $u$ is of class $C_{-\infty, \text { loc }}^{1,1}$ on $K \backslash\left(\partial_{\widetilde{M}} K \cup B\right)$ and that for almost all points $x=(z, w) \in K \backslash\left(\partial_{\widetilde{M}} K \cup B\right)$, Hess ${ }^{\mathrm{II}} u(x)$ is not of class $\left(-h_{x}^{+}\right)$. Then $\max u|K \leq \max u|\left(\partial_{\widetilde{M}} K \cup(K \cap B)\right)$.

Proof. Suppose that $\max u|K>\max u|\left(\partial_{\widetilde{M}} K \cup(K \cap B)\right)$. Then there is a compact set $K_{1} \subset K \cap \widetilde{M}$, such that $\max u\left|K_{1}>\max u\right| \partial_{\widetilde{M}} K_{1}$. By Lemma 3.7, there exists a function $v(z, w)$, satisfying condition (3.4) in a neighborhood of $K_{1}$, and $x_{0}=\left(z_{0}, w_{0}\right) \in \operatorname{Int} K_{1}$, such that $(u+v)\left(x_{0}\right)=0>(u+v)(x), x \in K_{1} \backslash\left\{x_{0}\right\}$. Since Hess ${ }^{\mathrm{II}} v \equiv 0, \operatorname{Hess}^{\mathrm{II}}(u+v)(x)$ does not belong to $-h_{x}^{+}$for a.a. $x \in \operatorname{Int}\left(K_{1}\right)$.

Choose now an admissible coordinate system $\left(\varsigma_{1}, \ldots, \varsigma_{k}, w_{1}, \ldots, w_{n}\right)$ defined in a neighborhood $U$ of $x_{0}$, and let $U^{\prime}$ denote the image of $U$ in $\mathbf{C}^{k+n}$ under the coordinate map. Denote by $u_{1}: U^{\prime} \rightarrow[-\infty,+\infty)$ the function corresponding to $(u+v)$ via this map. Then

$$
u_{1}\left(\varsigma_{0}, w_{0}\right)=0>u_{1}(\varsigma, w), \quad(\varsigma, w) \in U^{\prime} \backslash\left\{\left(\varsigma_{0}, w_{0}\right)\right\} .
$$

Furthermore, by the properties of an admissible coordinate system, $\operatorname{Hess}^{\mathrm{II}} u_{1}(\varsigma, w)$ is not of class $-h_{(\varsigma, w)}^{+}=-h^{+}$(it is now independent on $(\varsigma, w)$ ) for a.a. $(\varsigma, w) \in U^{\prime}$. 
This implies that $\operatorname{Hess}^{R} u_{1}(\zeta, w)$ is not negative definite at the same points. (If it were, then both its Hermitian part, $\operatorname{Hess}^{\mathbf{C}} u_{1}(\varsigma, w)$ would be negative definite, as well as the form $\left\langle\bar{W}, A_{22} W\right\rangle+\operatorname{Re}\langle W, B W\rangle$ (= the restriction of $\operatorname{Hess}^{\mathrm{R}} u_{1}(\zeta, w)$ to $\left.\{0\} \times \mathbf{C}^{n}\right)$. Then Hess ${ }^{\mathrm{II}} u(\varsigma, w) \in-h^{+}$, contrary to the assumption; cf. (3.5).) In addition, $u_{1} \in C_{-\infty, \text { loc }}^{1,1}\left(U^{\prime}\right)$. (Since $v$ is smooth, $(u+v) \in C_{-\infty, \text { loc }}^{1,1}$, and $u_{1} \in$ $C_{-\infty, \text { loc }}^{1,1}$ by Corollary (2.5).)

Concluding, $u_{1} \in C_{-\infty, \text { loc }}^{1,1}\left(U^{\prime}\right)$ and, for a.a. $(\varsigma, w) \in U^{\prime}$, $\operatorname{Hess}^{R} u_{1}(\varsigma, w)$ has at least one nonnegative eigenvalue. Hence, $u_{1}$ must have local maximum property on $U^{\prime}$, by the argument in [9, p. 319, lines 11-24]; cf. also [12, Theorem B.12]. This contradicts (3.7). Q.E.D.

LEMMA 3.8. Let $M$ be a complex manifold with the separation property. Let $P^{1}, P^{2}$ be classes of usc functions (nonnecessarily pseudoconvex or admissible) on $\widetilde{M}=M \times \mathbf{C}^{n}$, such that subclasses $C_{-\infty, \text { loc }}^{1,1} \cap P^{1}$ and $C_{-\infty, \text { loc }}^{1,1} \cap P^{2}$ are dense in $P^{1}$ and $P^{2}$ respectively, in the sense of Corollary 2.9. Let $Y_{x}^{1}, Y_{x}^{2}$ denote the classes of forms representing, at $x \in \widetilde{M}, P^{1}$ and $P^{2}$, respectively. Assume that $Y_{x}^{1} \subset\left(Y_{x}^{2}\right)^{D}$, for all $x \in \widetilde{M}$. Then $P^{1} \subset\left(P^{2}\right)^{d}$ and $P^{2} \subset\left(P^{1}\right)^{d}$.

ProOF. By [11, Definition 1.11], both statements are equivalent to showing that whenever $K \subset U \subset \widetilde{M}, u \in P^{1}(U), v \in P^{2}(U)$, where $U$ is open and $K$ compact, then

$$
\max _{K}(u+v) \leq \max _{\partial K}(u+v) .
$$

By the assumptions (cf. Corollary 2.9), there are functions

$$
u_{n} \in\left(C_{-\infty, 1 \text { loc }}^{1,1} \cap P^{1}\right)(\text { nbhd } K)
$$

and $v_{n} \in\left(C_{-\infty, \text { loc }}^{1,1} \cap P^{2}\right)($ nbhd $K), n=1,2, \ldots$, such that $u_{n}(x) \searrow u(x), v_{n}(x) \searrow$ $v(x), x \in K$.

Fix $n$. Then, for a.a. $x \in \operatorname{Int}(K)$, both $u_{n}$ and $v_{n}$ have the second-order Peano differential at $x$ and $\operatorname{Hess}^{\mathrm{II}}\left(u_{n}+v_{n}\right)(x) \in Y_{x}^{1}+Y_{x}^{2} \subset\left(Y_{x}^{2}\right)^{D}+Y_{x}^{2}$. By the definition of $\left(Y_{x}^{2}\right)^{D}$ (cf. Proposition 3.4 or [12, Definition A.2(iii)]), this implies that $\operatorname{Hess}^{\mathrm{II}}\left(u_{n}+v_{n}\right)(x)$ is not of class $-h_{x}^{+}$for a.a. $x \in \operatorname{Int}(K)$. By Lemma 3.7, $\max \left(u_{n}+v_{n}\right)\left|K \leq \max \left(u_{n}+v_{n}\right)\right| \partial K$. (Note that $\left.\left(u_{n}+v_{n}\right) \in C_{-\infty, \text { loc }}^{1,1}\right)$ This implies (3.8). Q.E.D.

We consider now an invariant admissible class $P$; cf. (1.8), (1.9), on a complex homogeneous space $\widetilde{M}=M \times \mathbf{C}^{n}, M=K / G$, and examine the relation between classes of forms $Y_{p}$ and $Y_{q}$ representing $P$ at two points $p$ and $q$ of $\widetilde{M}$.

Let $f: \widetilde{M} \rightarrow \widetilde{M}$ denote the action of any element of the group $\widetilde{K}=K \oplus$ $\mathbf{C}^{n}$, such that $f(p)=q$. Then $f$ is biholomorphic and its differential at $p, f_{*}=$ $\left(f_{*}\right)_{p}: T_{p}(\widetilde{M}) \rightarrow T_{q}(\widetilde{M})$, is a C-linear isomorphism.

If $u \in C_{-\infty, \text { loc }}^{1,1}$ (nbhd $q$ ) has the second-order Peano differential at $q$, then so does (at $p$ ) $u \circ f$. It follows from (3.2), (3.3) and computations similar to those in Krantz $\left[7\right.$, p. 105] that, if $F^{1}=\operatorname{Hess}^{\mathrm{II}}(u \circ f)(p)$ and $F=\operatorname{Hess}^{\mathrm{II}} u(q)$, then

$$
F^{1}(X, X)=F\left(f_{*}(X), f_{*}(X)\right), \quad X \in T_{p}(M) .
$$


(The reason for this is that $f$ acts separately in $z$ and $w$, is biholomorphic in $z$ and is just a translation in $w$.) If we denote $f^{*} F=F^{1}$, then we obtain an $R$-linear map

$$
f^{*}: h_{q} \rightarrow h_{p} .
$$

By the observations in the proof of Proposition 3.3, if $F \in h_{q}^{+}$(cf. Definition $3.1(\mathrm{~b}))$, then there is a smooth function $u(z, w)$, plurisubharmonic in $(z, w)$ and convex in $w$, such that $\operatorname{Hess}^{\mathrm{II}} u(q)=F$. By $(3.9), f^{*} F=\operatorname{Hess}^{\mathrm{II}}(u \circ f)(p)$. Since $u \circ f$ is clearly plurisubharmonic in $(z, w)$ and convex in $w, f^{*} F \in h_{p}^{+}$. Hence

$$
f^{*}\left(h_{q}^{+}\right) \subset h_{p}^{+} .
$$

Eventually, if $F \in Y_{q}^{\prime}$ (cf. Definition 3.1(c)), then $F=\operatorname{Hess}^{\mathrm{II}} u(q)$, for some function $u \in\left(C_{-\infty, \text { loc }}^{1,1} \cap P\right)($ nbhd $q)$. By $(3.9), f^{*} F=\operatorname{Hess}^{\mathrm{II}}(u \circ f)(p)$. Since $u \circ f \in P, f^{*} F \in Y_{p}^{\prime}$. Thus

$$
f^{*}\left(Y_{q}\right) \subset Y_{p}
$$

because $Y_{p}$ and $Y_{q}$ are closures of $Y_{p}^{\prime}$ and $Y_{q}^{\prime}$ respectively.

The same arguments applied to $f^{-1}$ yield the linear transformation $\left(f^{-1}\right)^{*}: h_{p}$ $\rightarrow h_{q}$ and inclusions $\left(f^{-1}\right)^{*}\left(h_{p}^{+}\right) \subset h_{q}^{+},\left(f^{-1}\right)^{*}\left(Y_{p}\right) \subset Y_{q}$. In addition, $\left(f^{-1}\right)^{*}=$ $\left(f^{*}\right)^{-1}$, by (3.9). By this and (3.10)-(3.12), get $f^{*}\left(h_{q}\right)=h_{p}, f^{*}\left(h_{q}^{+}\right)=h_{p}^{+}$and $f^{*}\left(Y_{q}\right)=Y_{p}$. We summarize these observations in the next corollary.

COROLLARY 3.9. Let $M=K / H$ be a complex homogeneous space and $P$ be an invariant admissible class of functions on $\widetilde{M}=M \times \mathbf{C}^{n}$. Let $g \in K \oplus \mathbf{C}^{n}, t_{g}: \widetilde{M} \rightarrow$ $\widetilde{M}$ be the action of $g$ on $\widetilde{M}, x \in \widetilde{M}$ and $y=t_{g} x$. Then $t_{g}^{*}: h_{y} \rightarrow h_{x}$ (defined by $\left.\left(t_{g}^{*} F\right)(X, X)=F\left(\left(t_{g}\right)_{*} X,\left(t_{g}\right)_{*} X\right), X \in T_{x}(\widetilde{M})\right)$ is an order isomorphism, i.e. $t_{g}^{*}\left(h_{y}^{+}\right)=h_{x}^{+}$and $t_{g}^{*}\left(Y_{y}\right)=Y_{x}$.

REMARK 3.10. In particular, $Y_{x}$ must be invariant with respect to the action of the isotropy group at $x$. This is in contrast with the Euclidean case [12, §3], where no such restriction occurs.

LEMMA 3.11. Under assumptions of Corollary 3.9, let $p \in \widetilde{M}=M \times \mathbf{C}^{n}$, $F \in Y_{p}$ and $F_{1} \in h_{p}^{+}$. Let $u \in C^{(2)}($ nbhd $p)$ and $\operatorname{Hess}^{\text {II }} u(p)=F+F_{1}$. Then

(i) $\operatorname{Hess}^{\mathrm{II}} u(x) \in \operatorname{Int}\left(Y_{x}\right)$ for $x$ near $p$;

(ii) $u \in P($ nbhd $p)$.

ProOF. (i) As observed in the proof of Lemma 2.6 (cf. (2.9), (2.10)), there is a neighborhood $V^{\prime}$ of $p$ in $\widetilde{M}$ and a smooth map $s: V^{\prime} \rightarrow \widetilde{K}=K \oplus \mathbf{C}^{n}$, such that

$$
\begin{gathered}
s(p)=e \oplus 0, \\
t_{s(x)} p=x, \quad x \in V^{\prime} .
\end{gathered}
$$

By Corollary 3.9,

$$
Y_{x}=\left(t_{s(x)}^{*}\right)^{-1}\left(Y_{p}\right) .
$$

Clearly, the function $x \rightarrow \operatorname{Hess}^{\mathrm{II}}\left(u \circ t_{s(x)}\right)(p): V^{\prime} \rightarrow h_{p}$ is continuous. Its value at $p$ is an interior point of $Y_{p}$, because $\operatorname{Hess}^{\mathrm{II}}\left(u \circ t_{s(p)}\right)(p)=F+F_{1} \subset Y_{p}+h_{p}^{+} \subset \operatorname{Int}\left(Y_{p}\right)$; cf. [12, Proposition A.5(iii)]. Hence, there is a neighborhood $V, p \in V \subset \widetilde{M}$, such 
that $\operatorname{Hess}^{\mathrm{II}}\left(u \circ t_{s(x)}\right)(p) \in \operatorname{Int}\left(Y_{p}\right)$ for $x \in V$. By this, (3.9) and Corollary 3.9, we get

$$
\operatorname{Hess}^{\mathrm{II}} u(x)=\left(t_{s(x)}^{*}\right)^{-1}\left(\operatorname{Hess}^{\mathrm{II}}\left(u \circ t_{s(x)}\right)(p)\right) \in\left(t_{s(x)}^{*}\right)^{-1}\left(\operatorname{Int} Y_{p}\right)=\operatorname{Int} Y_{x}
$$

This proves (i).

The proof of (ii) follows directly from (i) and the next assertion.

ASSERTION. If $V$ is an open set and $u: V \rightarrow R$ has the second-order Peano differential, such that $\operatorname{Hess}^{\mathrm{II}} u(x) \in \operatorname{Int}\left(Y_{x}\right)$ for every $x \in V$, then $u \in P(V)$.

To prove this it is enough, by [11, Theorem 3.5], to construct, for every $x \in V$, a neighborhood $V_{x}$ of $x$ and a continuous minorant $u^{x}$ of $u$, such that

$$
\begin{gathered}
u^{x} \in P\left(V_{x}\right), \quad u^{x}(x)=u(x) ; \\
u^{x}(y)<u(y), \quad \text { for } y \in V_{x} \backslash\{x\} .
\end{gathered}
$$

Recall that $h^{0}$, the convex cone of positive definite forms in $h_{x}$, is contained in $h_{x}^{+}$, and so $Y_{x}$ is an order cone with respect to the order structure $\left(h_{x}, h^{0}\right)$ as well. By this and [12, Proposition A.5(iii)], $\operatorname{Int}\left(Y_{x}\right)=Y_{x}+h^{0}=\left(Y_{x}+h^{0}\right)+h^{0}=\operatorname{Int}\left(Y_{x}\right)+h^{0}$, and so there are $F \in \operatorname{Int}\left(Y_{x}\right), F_{0} \in h^{0}$, such that $\operatorname{Hess}^{\mathrm{II}} u(x)=F+F_{0}$. Fix $x \in V$. By Proposition 3.3, $F \in Y_{x}^{\prime}$, i.e. $F=\operatorname{Hess}^{\mathrm{II}} u_{1}(x)$ for some function $u_{1} \in$ $\left(P \cap C_{-\infty, \text { loc }}^{1,1}\right)(\operatorname{nbhd} x)$, and so $\operatorname{Hess}^{\mathrm{II}}\left(u-u_{1}\right)=F_{0}$. Fix an admissible coordinate system at $x$. By Remark 3.2, there is a function $v$, satisfying condition (3.4), such that the second-order Peano differential of $u-u_{1}-v$ is equal to $\operatorname{Hess}^{\mathrm{II}}\left(u-u_{1}-v\right)=$ $\operatorname{Hess}^{\mathrm{II}}\left(u-u_{1}\right)$.

Let $u^{x}(y)=u_{1}(y)+v(y), y \in \operatorname{nbhd}(x)$. By axiom (1.8)(ii) and condition (3.4), $u^{x} \in P(\operatorname{nbhd} x)$. By adding a constant to $v$, if necessary, we obtain $u^{x}$ with $u^{x}(x)=u(x)$; cf. axiom (1.5). Thus (3.16) holds. Since $\left(u-u^{x}\right)(x)=0$, and the second-order Peano differential of $u-u^{x}$ at $x$ (with respect to fixed coordinate system) is equal to the positive definite form $A,\left(u-u^{x}\right)(y)>0$ for $y \neq x, y$ near $x$, which proves (3.17). Q.E.D.

COROLLARY 3.12. Let $M=K / H$ be a complex homogeneous space, $\widetilde{M}=M \times$ $\mathrm{C}^{n}$, and $P^{1}, P^{2}$ be two invariant admissible classes of functions on $\widetilde{M}$; $f$. (1.8)(ii), (1.9), with representing classes of forms at $x$ denoted by $Y_{x}^{1}$ and $Y_{x}^{2}$, respectively. Then $P=P^{1} \cap P^{2}$ is an invariant admissible class on $\widetilde{M}$ with representing class of forms at $x$, equal to $Y_{x}^{1} \cap Y_{x}^{2}$.

Proof. The invariance and admissibility of $P=P^{1} \cap P^{2}$ is obvious. Let $Y_{x}$ be the class of forms representing $P$ at $x$, with $Y_{x}^{\prime}$ as in Definition 3.1(c). Clearly, $Y_{x}^{\prime} \subset\left(Y_{x}^{1}\right) \cap\left(Y_{x}^{2}\right)$, and so

$$
Y_{x} \subset Y_{x}^{1} \cap Y_{x}^{2} .
$$

Let $F \in \operatorname{Int}\left(Y_{x}^{1}\right) \cap \operatorname{Int}\left(Y_{x}^{2}\right)$ and choose $u \in C^{(2)}($ nbhd $x)$ with Hess ${ }^{\mathrm{II}} u(x)=F$. By Lemma 3.1(ii), $u$ is both of class $P^{1}$ and $P^{2}$ near $x$, and so $u \in P(\operatorname{nbhd} x)$. Thus $F \in Y_{x}$ and, consequently, $\operatorname{Int}\left(Y_{x}^{1}\right) \cap \operatorname{Int}\left(Y_{x}^{2}\right) \subset Y_{x}$. By [12, Lemma A.10], $\mathrm{Cl}\left(\operatorname{Int}\left(Y_{x}^{1}\right) \cap \operatorname{Int}\left(Y_{x}^{2}\right)\right)=Y_{x}^{1} \cap Y_{x}^{2}$. Thus, $Y_{x}^{1} \cap Y_{x}^{2} \subset Y_{x}$ and, by (3.18), $Y_{x}^{1} \cap Y_{x}^{2}=Y_{x}$. Q.E.D. 


\section{Admissible classes of functions for complex interpolation of normed} spaces. Complex interpolation families of norms or quasi-norms on $\mathbf{C}^{n}$ parametrized by points of a complex manifold $M$ were defined in [13] in terms of a given pseudoconvex class of functions $P$ on $\widetilde{M}=M \times \mathbf{C}^{n}$. The natural requirement of complex theory that the construction be invariant with respect to biholomorphic bundle automorphisms

$$
(z, w) \rightarrow\left(z, T_{z}(w)\right): G \times \mathbf{C}^{n} \rightarrow G \times \mathbf{C}^{n},
$$

where $G \subset M$ and $T_{z}: \mathbf{C}^{n} \rightarrow \mathbf{C}^{n}$ are $\mathbf{C}$-affine and onto, leads to the following

DEFINITION 4.1. Let $M$ be a complex manifold (nonnecessarily a homogeneous space). Let $Q$ be a class of functions on $M$ and $R$ be a class on $\mathbf{C}^{n}$. We denote by $P_{Q}^{R}$ the class of all functions $u: U \rightarrow[-\infty,+\infty), U \subset \widetilde{M}=M \times \mathbf{C}^{n}$, such that the following two conditions hold:

$$
\begin{aligned}
& \text { whenever } f: V \rightarrow \mathbf{C}^{n}, V \subset M \text { is a holomorphic map, then the } \\
& \text { function } z \rightarrow u(z, f(z)) \text { is of class } Q \text { on }\{z \in V:(z, f(z)) \in U\} \text {; } \\
& \text { for every } z \text {, the slice function } w \rightarrow u(z, w) \text { is of class } R \text { (on } \\
& \{w:(z, w) \in U\}) \text {. }
\end{aligned}
$$

The next proposition can be checked in a straightforward fashion.

PROPOSITION 4.2. If $M$ is a complex manifold and classes $Q, R$ satisfy axioms (1.1)-(1.5) and (1.7), then so does the class $P_{Q}^{R}$.

In the abstract setting of [11, Definition 1.4], it is not obvious whether pseudoconvexity of $Q$ and $R$ implies that of $P_{Q}^{R}$, especially whether the abstract version of the axiom (1.9) holds; cf. [11, Condition (1.9)(i),(ii)]. It holds, however, in the invariant situation studied here.

Proposition 4.3. Let $M=K / H$ be a complex homogeneous space. Let $Q$ be an invariant complex pseudoconvex class on $M$ (i.e. conditions (1.1)-(1.7), (1.8)(i), (1.9) hold) and let $R$ be a translation invariant pseudoconvex class on $\mathbf{C}^{n}$. Assume that $R$ contains constant functions. Then $P_{Q}^{R}$ is an invariant admissible pseudoconvex class of functions on $\widetilde{M}=M \times \mathbf{C}^{n}$ (i.e. conditions (1.1)-(1.7), (1.8)(ii) and (1.9) hold).

PROOF. Condition (1.9) follows immediately from Definition 4.1 and properties of $Q$ and $R$.

Admissibility. Recall that, by definition (cf. $[\mathbf{1 1},(0.1)])$, a translation invariant pseudoconvex class on $\mathbf{C}^{n}$ satisfies the following condition:

$$
\begin{aligned}
& \text { if } u \in R(G), G \subset \mathbf{C}^{n} \text { and } v: G \rightarrow R \text { is locally convex on } G \text {, then } \\
& (u+v) \in R(G) .
\end{aligned}
$$

Thus, if $u \in P_{Q}^{R}(U)$ and $v(z, w)$ is locally convex in $w$, then the slice functions $w \rightarrow(u+v)(z, w)$ are of the class $R$, for every $z$. If $v(z, w)$ is, in addition, plurisubharmonic, $u \in P_{Q}^{R}$ and $f: V \rightarrow \mathbf{C}^{n}$ is holomorphic, then the function $z \rightarrow u(z, f(z))$ is of class $Q$, function $z \rightarrow v(z, f(z))$ is plurisubharmonic, and so $z \rightarrow(u+v)(z, f(z))$ is of class $Q$, by $(1.8)(\mathrm{i})$. Hence, $(u+v) \in P_{Q}^{R}(U)$, and so condition (1.8)(ii) holds. 
Condition (1.6). If $U \subset \widetilde{M}$ is relatively compact, choose a relatively compact open set $V \subset M$, such that $U \subset V \times \mathbf{C}^{n}$. By (1.6), there is a bounded function $u_{0} \in Q(V)$. Let $u(z, w)=u_{0}(z)$. Clearly, $u$ is a bounded function of class $P_{Q}^{R}$ on $U$. (This is the only place in the proof, where the property that constant functions belong to $R$ is used.)

The remaining conditions for the pseudoconvexity of $P_{Q}^{R}$ were established in Proposition 4.2. Q.E.D.

The next observation is a direct consequence of Definition 4.1.

PROPOSITION 4.4. If, in the situation of Definition 4.1, class $R$ is invariant with respect to the action of the group $\mathrm{Gl}\left(\mathbf{C}^{n}\right)$, then the class $P_{Q}^{R}$ is closed with respect to the composition with biholomorphic bundle automorphisms (4.1).

In the remainder of this section, we will restrict our attention to the following special cases of $\mathrm{Gl}\left(\mathbf{C}^{n}\right)$ invariant classes: $R=$ co, the class of all (locally) convex functions on (open subsets) of $\mathbf{C}^{n} ; R=\mathrm{psh}$, the class of all plurisubharmonic functions on $\mathbf{C}^{n}$, and the trivial case $R=$ usc, the class of all upper-semicontinuous functions.

Notation 4.5. By $P_{Q}^{\mathrm{co}}, P_{Q}^{\mathrm{psh}}$ and $P_{Q}$ we mean the class $P_{Q}^{R}$ with $R$ corresponding to co, psh and usc, respectively. By (usc) ${ }^{R}$ we denote the class of all usc functions $u(z, w)$ on $M \times \mathbf{C}^{n}$, such that all the slice functions $w \rightarrow u(z, w)$ are of class $R$.

The following is obvious.

Proposition 4.6. $P_{Q}^{R}=P_{Q} \cap(\text { usc })^{R}$.

The following classes $Q$ are most important for complex interpolation of normed spaces in [13]: $Q=$ the class of all functions that are subharmonic with respect to a given invariant Kähler metric on $M ; Q=P_{q}=$ the class of all $q$-plurisubharmonic functions on $M$, where $0 \leq q \leq k-1, k=\operatorname{dim}_{\mathrm{C}} M$; cf. [5, 9].

PROPOSITION 4.7. If $M$ is a complex manifold and $Q=P_{q}$ (on $M$ ), then $P_{Q}=P_{q}($ on $\widetilde{M})$. Furthermore $P_{Q}^{R}=P_{q} \cap(\text { usc })^{R}$.

REMARK 4.8. Consistently with Proposition 4.7, we let $P_{q}^{\text {co }}, P_{q}^{\text {psh }}$ stand for the classes $P_{Q}^{\mathrm{co}}, P_{Q}^{\mathrm{psh}}$, on $M \times \mathbf{C}^{n}$, where $Q=P_{q}$ (on $\left.M\right)$. Clearly,

$$
\begin{aligned}
P_{q}^{\mathrm{co}} & =P_{q} \cap(\mathrm{usc})^{\mathrm{co}}, \\
P_{q}^{\mathrm{psh}} & =P_{q} \cap(\mathrm{usc})^{\mathrm{psh}} .
\end{aligned}
$$

ProOF OF PROPOSITION 4.7. Since the class $P_{q}$ is biholomorphically invariant [10, Property 1.11], we can assume without loss of generality that $M=\mathrm{C}^{k}$. The class $P_{Q}=P_{Q}^{\text {usc }}$, with $Q=P_{q}$ on $\mathbf{C}^{k}$, is a translation invariant pseudoconvex class on $\mathbf{C}^{k} \times \mathbf{C}^{n}$ (cf. Proposition 4.3), and so the class $C \cap P_{Q}$ (where $C=$ the class of all continuous functions) is dense in $P_{Q}$ in the sense of Corollary 2.9. Hence, it is enough to show that, whenever $u \in\left(C \cap P_{Q}\right)(U), U \subset \mathbf{C}^{k+n}$, then $u \in P_{q}(U)$.

By the definition, we have to check (cf. Hunt and Murray [5], or $[\mathbf{9}, \S 1]$ ) that, for every complex hyperplane $L \subset \mathbf{C}^{k+n}$, with $\operatorname{dim}_{\mathbf{C}} L=q+1$, the restriction $u \mid L \cap U$ is $q$-plurisubharmonic. Since $q \leq k-1$, it clearly suffices to do the same for every hyperplane $L_{0}$ with $\operatorname{dim}_{\mathrm{C}} L_{0}=k$. In case $L_{0}$ does not contain any 
vertical (i.e. $w$-direction), $L_{0}$ can be represented as $L_{0}=\left\{\left(z, t_{0}(z)\right): z \in \mathbf{C}^{k}\right\}$, where $t_{0}: \mathbf{C}^{k} \rightarrow \mathbf{C}^{n}$ is a $\mathbf{C}$-affine map. Then $u \mid L_{0} \cap U \in P_{q}$, because the function $z \rightarrow u\left(z, t_{0}(z)\right)$ is of class $Q=P_{q}$ (on $\mathbf{C}^{k}$ ), by condition (4.2). In case $L_{0}$ contains a vertical direction, choose a sequence of complex hyperplanes $L_{j}$, such that $L_{j} \rightarrow L_{0}$ in the gap topology and none of $L_{j}$ 's, $j=1,2, \ldots$, contains any vertical direction. Fix $\left(z_{0}, w_{0}\right) \in U$. It is easy to see that there are $\mathbf{C}$-affine maps $T_{j}: \mathbf{C}^{k} \rightarrow \mathbf{C}^{k+n}$, $j=0,1,2, \ldots$, such that $T_{j}\left(\mathbf{C}^{k}\right)=L_{j}, j=0,1,2, \ldots ; T_{0}(0)=\left(z_{0}, w_{0}\right)$ and $T_{j} \rightarrow T_{0}$, uniformly on compact sets. Since $L_{j}$ are nonvertical, $u \mid L_{j} \cap U$ is $Q$ plurisubharmonic (as it was already observed), and so $u \circ T_{j} \in P_{q}$, by [9, Property 1.11]. Since $u$ is continuous, $u \circ T_{j}(z) \rightarrow u \circ T_{0}(z)$, uniformly on a neighborhood of $0 \in \mathbf{C}^{k}$, and so $u \circ T_{0} \in P_{q}$, near 0 . (This easy fact follows, for example, from [11, Proposition 3.2].) Since $T_{0}$ is biholomorphic, $u_{0} \mid L_{0}$ is $q$-plurisubharmonic near $\left(z_{0}, w_{0}\right)$. Q.E.D.

THEOREM 4.9. Let $M=K / H$ be a complex homogeneous space and let $Q$ be a complex, invariant pseudoconvex class on $M$ (i.e. (1.1)-(1.7), (1.8)(i) and (1.9) hold), satisfying the condition

$$
\text { whenever } g \in Q \text { and } r>0 \text {, then } r g \in Q \text {. }
$$

Let $\varphi \in C^{(2)}(R)$ have positive derivative on $R$ and $u(z, w)$ be a usc function on $U \subset \widetilde{M}=M \times \mathbf{C}^{n}$. Then

(i) the function $\varphi \circ u \in\left(P_{Q}^{c o}\right)^{d}$ (cf. [11, Definition 1.11]), provided the function $(z, w) \rightarrow \varphi(g(z)+u(z, w))$ is of class $\left(P_{0}^{\text {co }}\right)^{d}$ for every $g \in Q \cap C_{-\infty, \mathrm{loc}}^{1,1}$,

(ii) $\varphi \circ u$ is of class $\left(P_{Q}^{\mathrm{psh}}\right)^{d}$, provided for every $g \in Q \cap C_{-\infty, \text { loc }}^{1,1}$ the function $(z, w) \rightarrow \varphi(g(z)+u(z, w))$ is of class $\left(P_{0}\right)^{d}=P_{k+n-1}$.

(iii) if the function $\varphi(h(z)+u(z, w))$ is of class $\left(P_{Q}^{\text {co }}\right)^{d}$ (or of class $\left.\left(P_{Q}^{\mathrm{psh}}\right)^{d}\right)$, for every pluriharmonic function $h(z)$ on $M$, then, for every $g \in Q$, the function $\varphi(g(z)+u(z, w))$ is of class $\left(P_{0}^{\mathrm{co}}\right)^{d}$ (or of class $\left(P_{0}\right)^{d}=P_{k+n-1}$ respectively).

REMARK 4.10. As an examination of the proof of Theorem 4.9 shows, in case $\varphi(x)=x$, condition (4.7) is not needed.

To prove the theorem, we will identify first (in Lemmas 4.11 and 4.12) the classes of quadratic forms representing $P_{Q}^{\text {co }}$ and $P_{Q}^{\text {psh }}$ at $p \in \widetilde{M}$, as well as their dual order cones. The description will be in terms of the representing class of forms for $Q$.

We will use the formulism introduced at the beginning of $\S 3$; cf. (3.1)-(3.5) and remarks proceeding Definition 3.1. In particular, we fix $p=(a, b) \in \widetilde{M}=$ $M \times \mathbf{C}^{n}$ and an admissible coordinate system $\left(\varsigma_{1}, \ldots, \varsigma_{k}, w_{1}, \ldots, w_{n}\right)$ near $p$, and use routinely the following block representation (relative to the fixed coordinate system) of forms of type II, i.e. $F \in h_{p}$ :

$$
\begin{aligned}
F(Z \oplus W) & =A(Z \oplus W)+\operatorname{Re}\langle W, B W\rangle \\
& =\left\langle\bar{Z}, A_{11} Z\right\rangle+\left\langle\bar{Z}, A_{12} W\right\rangle+\left\langle\bar{W}, A_{21} Z\right\rangle+\left\langle\bar{W}, A_{22} W\right\rangle+\operatorname{Re}\langle W, B W\rangle,
\end{aligned}
$$

where $A_{11}, A_{12}, A_{21}, A_{22}$ and $B$ are matrices of sizes $k \times k, k \times n, n \times k, n \times n$ and $n \times n$ respectively, and $A_{i j}^{*}=A_{j i}, i, j=1,2$, and $B^{T}=B$.

Note also that, since $Q$ is a complex class (cf. (1.8)(i)), the situation in Definition 3.1 simplifies as follows: $\operatorname{Hess}^{\text {II }} u=\operatorname{Hess}^{C} u$ for $u \in C_{-\infty, \text { loc }}^{1,1}(M), h_{p}$ reduces to the 
space of Hermitian forms on $T_{p}(M)$ and the cone $h_{p}^{+}$is identical with the cone $h^{0}$ of positive definite forms in $h_{p}$. Representation (3.5) reduces to $\langle\bar{Z}, A Z\rangle, A=A^{*}$, and the representing class $Y$ for $Q$ has the following properties; cf. [12, Definition A.2(iii)]:

$$
\begin{aligned}
& Y+A \subset Y, \text { if } A \text { is positive definite; } \\
& Y^{D}=\{A: Y+A \text { does not contain any negative definite form }\} .
\end{aligned}
$$

LEMMA 4.11. Let $M=K / H$ and $Q$ be as in Theorem 4.9. Let $p=(a, b) \in$ $\widetilde{M}=M \times \mathbf{C}^{n}$ and let $Y, Y^{\mathrm{co}}, Y^{\mathrm{psh}}$ denote classes of forms representing pseudoconvex classes $Q, P_{Q}^{\text {co }}$ and $P_{Q}^{\mathrm{psh}}$ at points $a, p$ and $p$ respectively. Then

(i) the form (4.8) belongs to $Y^{\mathrm{psh}}$ if and only if $A_{22}$ is positive semidefinite and the following condition holds:

$$
\left(A_{11}+A_{12} S+S^{*} A_{21}+S^{*} A_{22} S\right) \in Y \quad \text { for every } S \in \mathbf{C}[n \times k] ;
$$
$\in Y$;

(ii) if $A_{22}$ is positive definite, then (4.10) holds if and only if $\left(A_{11}-A_{12} A_{22}^{-1} A_{21}\right)$

(iii) the form (4.8) belongs to $Y^{\mathrm{co}}$ if and only if $A_{22}$ is positive semidefinite, condition (4.10) holds, and

$$
|\langle W, B W\rangle| \leq\left|\left\langle\bar{W}, A_{22} W\right\rangle\right| \text { for } W \in \mathbf{C}^{n} .
$$

ProOF. Let $\widetilde{Y}, Z^{\text {co }}$ and $Z^{\text {psh }}$ be classes of forms representing, at $p \in \widetilde{M}$, invariant and admissible pseudoconvex classes $P_{Q}$, (usc) $^{\text {co }}$ and (usc) ${ }^{\text {psh }}$, respectively. Obvious relations $P_{Q}^{\mathrm{co}}=P_{Q} \cap(\mathrm{usc})^{\mathrm{co}}$ and $P_{Q}^{\mathrm{psh}}=P_{Q} \cap(\mathrm{usc})^{\mathrm{psh}}$, and Corollary 3.12 imply that

$$
\begin{aligned}
Y^{\mathrm{co}} & =\tilde{Y} \cap Z^{\mathrm{co}}, \\
Y^{\mathrm{psh}} & =\tilde{Y} \cap Z^{\mathrm{psh}} .
\end{aligned}
$$

Since (usc) ${ }^{\mathrm{co}}$ and (usc) $)^{\mathrm{psh}}$ consist precisely of functions $u(z, w)$ that are convex or plurisubharmonic in $w$, and usc otherwise, the following observations are clear:

$$
\text { form (4.8) belongs to } Z^{\text {psh }} \text { if and only if } A_{22} \text { is positive semidefinite; }
$$

form (4.8) belongs to $Z^{\text {co }}$ if and only if the form $\left\langle\bar{W}, A_{22} W\right\rangle+$ $\operatorname{Re}\langle W, B W\rangle$ is positive semidefinite, which is equivalent to condition (4.11).

Relations (4.12)-(4.15) imply clearly that in order to prove parts (i) and (iii) of the lemma, it suffices to establish the next two assertions.

ASSERTION 1. If the form (4.8) belongs to $\widetilde{Y}$, then condition (4.10) holds.

ASSERTION 2. If the block component $A_{22}$ is positive semidefinite and condition (4.10) holds, then the form (4.8) belongs to $\widetilde{Y}$.

As for part (ii) of the lemma, it follows from the following matrix identity (which holds, if $A_{22}$ is positive definite):

$$
\begin{aligned}
& \left\langle\bar{Z},\left(A_{11}+A_{12} S+S^{*} A_{21}+S^{*} A_{22} S\right) Z\right\rangle \\
& \quad=\left\langle\bar{Z},\left(A_{11}-A_{12} A_{22}^{-1} A_{21}\right) Z\right\rangle+\left|\left(A_{22}^{-1 / 2} A_{21}+A_{22}^{1 / 2} S\right) Z\right|^{2} .
\end{aligned}
$$


(Indeed, if (4.10) holds, then using $S$, such that $A_{22}^{-1 / 2} A_{21}+A_{22}^{1 / 2} S=0$, we get $\left(A_{11}-A_{12} A_{22}^{-1} A_{21}\right) \in Y$. Conversely, if the latter form is of class $Y$, then its sum with the positive semidefinite Hermitian form $\left|\left(A_{22}^{-1 / 2} A_{21}+A^{1 / 2} S\right) Z\right|^{2}$ is also of class $Y$, by Proposition 3.3, and so (4.10) holds by the identity (4.16). This proves part (ii).)

ProOF OF ASSERTION 1. By Definition 3.1(c), $\tilde{Y}$ is the closure in $h_{p}$ of the set of all forms $\operatorname{Hess}^{\mathrm{II}} u(p)$, where $u \in P_{Q} \cap C_{-\infty, \text { loc }}^{1,1}$, and so we can assume without loss of generality that the form (4.8) is equal to $\operatorname{Hess}^{\mathrm{II}} u(p), u \in P_{Q} \cap C_{-\infty, \mathrm{loc}}^{1,1}$. Let $s \in \mathbf{C}[n \times k]$. We can also assume that the fixed admissible coordinate system $\left(\varsigma_{1}, \ldots, \varsigma_{k}, w_{1}, \ldots, w_{n}\right)$ has the property that $\varsigma(a)=0 \in \mathbf{C}^{k}$. Define the map $f(z)=b+S\left[\varsigma_{1}(z), \ldots, \zeta_{k}(z)\right]^{T}$. Clearly, $f: V \rightarrow \mathbf{C}^{n}$, where $V$ is some neighborhood of $a$, is analytic, and by condition (4.2), the function $v(z)=u(z, f(z))$ is of class $Q(V)$. Thus, $\operatorname{Hess}^{\mathbf{C}} v(a) \in Y$. (Function $v \in C_{-\infty, 10 c}^{1,1}$ and has the second-order Peano differential at $a$.) One can check that Hess ${ }^{C} v(a)$ is the form

$$
\left\langle\bar{Z},\left(A_{11}+A_{12} S+S^{*} A_{21}+S^{*} A_{22} S\right) Z\right\rangle
$$

and so condition (4.10) is established.

PROOF OF ASSERTION 2. Observe first that any form (4.8), such that $A_{22}$ is positive semidefinite and condition (4.10) holds, belongs to the closure, in $h_{p}$, of the set of all forms (4.8), such that

$$
\begin{aligned}
& A_{22} \text { is positive definite, }\left(A_{11}-A_{12} A_{22}^{-1} A_{21}\right) \in \operatorname{Int} Y \text { and, for every } \\
& S \in \mathrm{C}[n \times k], \text { the form } A_{11}+A_{12} S+S^{*} A_{21}+S^{*} A_{22} S \text { belongs to } \\
& Y .
\end{aligned}
$$

Indeed, if $F(Z \oplus W)$ (cf. (4.8)) is the original form, then the forms $F(Z \oplus W)+$ $\varepsilon|Z|^{2}+\varepsilon|W|^{2}, \varepsilon>0$, are the desired approximations. First, condition (4.10) holds for such an approximation because $\left(A_{11}+\varepsilon I_{k}\right)+A_{12} S+S^{*} A_{21}+S^{*}\left(A_{22}+\varepsilon I_{n}\right) S=$ $\left(A_{11}+A_{12} S+S^{*} A_{21}+S^{*} A_{22} S\right)+\varepsilon\left(I_{k}+S^{*} S\right)$. This form is the sum of a form of class $Y$ and of the positive semidefinite form $\varepsilon\left(I_{k}+S^{*} S\right)$, and so of class $Y$ itself. The same conclusion holds for the form $F(Z \oplus W)+\varepsilon|W|^{2}$, by the same argument. Applying the equivalence (ii) to the latter form we obtain that the Schur factor $A_{11}-A_{12}\left(A_{22}+\varepsilon I_{n}\right)^{-1} A_{21}$ is of class $Y$. Now, the analogous Schur factor for the form $F(Z \oplus W)+\varepsilon|Z|^{2}+\varepsilon|W|^{2}$ is equal to $\varepsilon I_{k}+\left[A_{11}-A_{12}\left(A_{22}+\varepsilon I_{n}\right)^{-1} A_{21}\right]$, and so it belongs to the class $\varepsilon I_{k}+Y$. By [12, Proposition A.5(iii)], the latter class is contained in $\operatorname{Int}(Y)$, and so is the Schur factor.

By the above argument, it suffices to show that any form (4.8), satisfying condition (4.17), belongs to $\tilde{Y}$. By an elementary matrix computation, such form can be represented as follows:

$$
\begin{aligned}
F(Z \oplus W)= & \left\langle\bar{Z},\left(A_{11}-A_{12} A_{22}^{-1} A_{21}\right) Z\right\rangle+\left|A_{22}^{-1 / 2} A_{21} Z+A_{22}^{1 / 2} W\right|^{2} \\
& +\operatorname{Re}\langle W, B W\rangle .
\end{aligned}
$$

Since $\left(A_{11}-A_{12} A_{22}^{-1} A_{21}\right) \in \operatorname{Int} Y$, we can apply Corollary 3.12 and obtain a smooth function $u_{0} \in Q($ nbhd $a)$, such that

$$
\operatorname{Hess}^{\mathrm{C}} u_{0}(a)=A_{11}-A_{12} A_{22}^{-1} A_{21} .
$$


Using the fixed admissible coordinate system $\varsigma_{1}, \varsigma_{2}, \ldots, \varsigma_{k}, w_{1}, \ldots, w_{n}$ at $p$, we define now

$$
v(z, w)=\left|A_{22}^{-1 / 2} A_{21} \varsigma(z)+A_{22}^{1 / 2} w\right|^{2}+\operatorname{Re}\langle w, B w\rangle .
$$

Clearly, $v$ is a plurisubharmonic function in $(z, w)$ in a neighborhood of $p$. It follows immediately from condition (4.2) of Definition 4.1 that the function $u(z, w):=$ $u_{0}(z)+v(z, w)$ is of class $P_{Q}$ (note that $Q$ is a complex class; cf. (1.8)(i)). By (4.18)-(4.20), Hess ${ }^{\text {II }} u(p)=F$, the given form satisfying (4.17). Thus, $F \in \widetilde{Y}$, which completes the proof of Assertion 2. Q.E.D.

LEMMA 4.12. If $M=K / H$ and $Q$ satisfy the assumptions of Theorem 4.9, and $Y, Y^{\mathrm{co}}, Y^{\mathrm{psh}}$ are defined as in Lemma 4.11, then:

(i) form (4.8) belongs to $\left(Y^{\mathrm{psh}}\right)^{D}$ if and only if $A_{22}$ is not negative definite or the following condition holds:

$$
\left(A_{11}+A_{12} S+S^{*} A_{21}+S^{*} A_{22} S\right) \in Y^{D} \quad \text { for some } S \in \mathbf{C}[n \times k] ;
$$

(ii) if $A_{22}$ is negative definite, then condition (4.21) holds if and only if $\left(A_{11}-A_{12} A_{22}^{-1} A_{21}\right) \in Y^{D}$;

(iii) form (4.8) belongs to $\left(Y^{\mathrm{co}}\right)^{D}$ if and only if the form $\left\langle\bar{W}, A_{22} W\right\rangle+\operatorname{Re}\langle W, B W\rangle$ is not negative definite or condition (4.21) holds.

PROOF. As in the last proof, the lemma will follow from properties of $Y$, the class of forms representing $P_{Q}$ at $p$.

ASSERTION 1. If form (4.8) satisfies condition (4.21), then it belongs to $\tilde{Y}^{D}$.

If $F$ is the form under consideration, with block components $A_{i j}, B$, choose $S_{0} \in \mathbf{C}[n \times k]$, such that

$$
\left(A_{11}+A_{12} S_{0}+S_{0}^{*} A_{21}+S_{0}^{*} A_{22} S_{0}\right) \in Y^{D} .
$$

Consider an arbitrary form $F^{\prime} \in \widetilde{Y}$, with block components $A_{i j}^{\prime}, B^{\prime}$ in the representation (4.8). By Assertion 1 in the proof of Lemma 4.11, applied to $S=S_{0}$, we get $\left(A_{11}^{\prime}+A_{12}^{\prime} S_{0}+S_{0}^{*} A_{21}^{\prime}+S_{0}^{*} A_{22}^{\prime} S_{0}\right) \in Y$. This and the relation (4.22) implies that the form

$$
\left(A_{11}+A_{11}^{\prime}\right)+\left(A_{12}+A_{12}^{\prime}\right) S_{0}+S_{0}^{*}\left(A_{21}+A_{21}^{\prime}\right)+S_{0}^{*}\left(A_{22}+A_{22}^{\prime}\right) S_{0}
$$

belongs to $Y^{D}+Y$, and so it cannot be a negative definite form on $T_{p}(M)$. This means that the Hermitian part of the form $F+F^{\prime}$ cannot be negative definite, and so the form $F+F^{\prime}$ does not belong to $\left(-h_{p}^{+}\right)$, provided $F^{\prime} \in \widetilde{Y}$. This proves the assertion.

ASSERTION 2. If $F$ is a form with representation (4.8) and $A_{22}$ is negative definite, then the following conditions are equivalent:

(a) $F \in \tilde{Y}^{D}$;

(b) $\left(A_{11}+A_{12} S+S^{*} A_{21}+S^{*} A_{22} S\right) \in Y^{D}$ for some $S \in \mathrm{C}[n \times k]$;

(c) $\left(A_{11}-A_{12} A_{22}^{-1} A_{21}\right) \in Y^{D}$. 
We prove it using the following matrix identities, which are valid when $A_{22}$ is negative definite:

$$
\begin{aligned}
& \left\langle\bar{Z},\left(A_{11}+A_{12} S+S^{*} A_{21}+S^{*} A_{22} S\right) Z\right\rangle \\
& \quad=\left\langle\bar{Z},\left(A_{11}-A_{12} A_{22}^{-1} A_{21}\right) Z\right\rangle-\left|\left(-A_{22}\right)^{-1 / 2} A_{21} Z+\left(-A_{22}\right)^{1 / 2} S Z\right|^{2}
\end{aligned}
$$

$$
\begin{aligned}
F(Z \oplus W)= & \left\langle\bar{Z},\left(A_{11}-A_{12} A_{22}^{-1} A_{21}\right) Z\right\rangle-\left|\left(-A_{22}\right)^{-1 / 2} A_{21} Z+\left(-A_{22}\right)^{1 / 2} W\right|^{2} \\
& +\operatorname{Re}\langle W, B W\rangle .
\end{aligned}
$$

We note that the implication $(\mathrm{b}) \Rightarrow(\mathrm{a})$ constitutes Assertion 1 .

(a) $\Rightarrow$ (c). Suppose that $\left(A_{11}-A_{12} A_{22}^{-1} A_{21}\right) \notin Y^{D}$. Then, by (4.9), there is $A_{11}^{\prime} \in Y$ and $\varepsilon>0$, such that

$$
\left\langle\bar{Z}, A_{11}^{\prime} Z\right\rangle+\left\langle\bar{Z},\left(A_{11}-A_{12} A_{22}^{-1} A_{21}\right) Z\right\rangle \leq-\varepsilon|Z|^{2}, \quad Z \in \mathbf{C}^{k} .
$$

By Assertion 2 in the proof of Lemma 4.11, the form $\left\langle\bar{Z}, A_{11}^{\prime} Z\right\rangle-\operatorname{Re}\langle W, B W\rangle$, which has block components $A_{11}^{\prime}, 0,0,0,-B$, belongs to $\tilde{Y}$. By (4.24) and (4.25), $F(Z \oplus W)+\left\langle\bar{Z}, A_{11}^{\prime} Z\right\rangle-\operatorname{Re}\langle W, B W\rangle \leq-\varepsilon|Z|^{2}-\left|\left(-A_{22}\right)^{-1 / 2} A_{21} Z+\left(-A_{22}\right)^{1 / 2} W\right|^{2} ;$

the latter form is negative definite because $A_{22}$ is nonsingular. Consequently, $F(Z \oplus$ $W)+\left\langle\bar{Z}, A_{11}^{\prime} Z\right\rangle-\operatorname{Re}\langle W, B W\rangle$ is a negative definite form, and so of class $\left(-h_{p}^{+}\right)$, which belongs to $\tilde{Y}^{D}+\tilde{Y}$. This is a contradiction.

(c) $\Rightarrow$ (b). Substituting $S=-\left(-A_{22}\right)^{-1} A_{21}$ into (4.23), we get

$$
\left\langle\bar{Z},\left(A_{11}+A_{12} S+S^{*} A_{21}+S^{*} A_{22} S\right) Z\right\rangle=\left\langle\bar{Z},\left(A_{11}-A_{12} A_{22}^{-1} A_{21}\right) Z\right\rangle,
$$

which is of class $Y^{D}$. The proof of Assertion 2 is complete.

Recall that $Y^{\mathrm{psh}}=\widetilde{Y} \cap Z^{\mathrm{psh}}, Y^{\mathrm{co}}=\widetilde{Y} \cap Z^{\mathrm{co}}$, by (4.12), (4.13). This and [12, Corollary A.11(ii)] imply that

$$
\begin{aligned}
\left(Y^{\mathrm{psh}}\right)^{D} & =(\widetilde{Y})^{D} \cup\left(Z^{\mathrm{psh}}\right)^{D}, \\
\left(Y^{\mathrm{co}}\right)^{D} & =(\tilde{Y})^{D} \cup\left(Z^{\mathrm{co}}\right)^{D} .
\end{aligned}
$$

On the other hand, by (4.14) and (4.15), the next two observations are obvious.

(4.28) Form (4.8) belongs to $\left(Z^{\mathrm{psh}}\right)^{D}$ if and only if $A_{22}$ is not negative definite.

(4.29) Form (4.8) belongs to $\left(Z^{\text {co }}\right)^{D}$ if and only if the symmetric form $\left\langle\bar{W}, A_{22} W\right\rangle+\operatorname{Re}\langle W, B W\rangle$ is not negative definite.

In particular, if form (4.8) is not in $\left(Z^{\mathrm{psh}}\right)^{D}$ or $\left(Z^{\mathrm{co}}\right)^{D}$, then $A_{22}$ is negative definite and Assertion 2 applies. The lemma follows directly from the assertions and relations (4.26)-(4.29). Q.E.D.

LEMMA 4.13. If form (4.8) belongs to the class of forms representing $\left(P_{0}^{\mathrm{co}}\right)^{d}$ at $p=(a, b) \in \widetilde{M}$, then at least one of the following two forms is not negative definite:

$$
\begin{gathered}
\left\langle\bar{Z}, A_{11} Z\right\rangle+\left\langle\bar{Z}, A_{12} W\right\rangle+\left\langle\bar{W}, A_{21} Z\right\rangle+\left\langle\bar{W}, A_{22} W\right\rangle ; \\
\left\langle\bar{W}, A_{22} W\right\rangle+\operatorname{Re}\langle W, B W\rangle .
\end{gathered}
$$


ProOF. Denote by $Y_{0}^{\text {co }}$ and $Y_{0}$ the classes of forms representing respectively pseudoconvex classes $P_{0}^{\mathrm{co}}$ and $P_{0}$ at $p$ (cf. Remark 4.8). Since $P_{0}^{\mathrm{co}}=P_{0} \cap$ (usc) (co $^{\mathrm{co}}$, Corollary 3.12 implies that $Y_{0}^{\mathrm{co}}=Y_{0} \cap Z^{\mathrm{co}}$ (cf. (4.15) and preceding remarks) and so, by $[12$, Corollary A.11(ii)],

$$
\left(Y_{0}^{\mathrm{co}}\right)^{D}=Y_{0}^{D} \cup\left(Z^{\mathrm{co}}\right)^{D} .
$$

It is clear that a form $F$ represented by (4.8) belongs to $Y_{0}^{D}$ if and only if form (4.30) is not negative definite. By this and relations (4.29), (4.32), the set $\left(Y_{0}^{\mathrm{co}}\right)^{D}$ consists precisely of form $F$, such that at least one of the forms (4.30), (4.31) is not negative definite. This implies the lemma by Proposition 3.4. Q.E.D.

ProOF OF THEOREM 4.9(i). Fix function $\varphi: R \rightarrow R$ with required properties. Our basic strategy is to apply Lemma 3.8 to classes $P^{1}, P^{2}$ of usc functions (on $\widetilde{M}$ ) defined as follows.

We define $P^{1}$ as the set of all usc functions $U(z, w)=\varphi(u(z, w))$ on $\widetilde{M}$, such that

$$
\text { for every } g \in C^{(2)} \cap Q \text {, the function }(z, w) \rightarrow \varphi(g(z)+u(z, w)) \text { is }
$$
of class $\left(P^{\mathrm{co}}\right)^{d}$,

and let $P^{2}=P_{Q}^{\text {co }}$. By Proposition $4.3, P^{2}$ is an invariant, admissible pseudoconvex class on $\widetilde{M}$, and so $P^{2} \cap C_{-\infty, \text { loc }}^{1,1}$ is dense in $P^{2}$ in the sense of Corollary 2.9, as required in Lemma 3.8. It is less obvious that class $P^{1}$ has the same property, because this class is apparently not a generalized pseudoconvex one (namely, axiom (1.5) is unlikely to hold). Consider, however, the class of all usc functions $u(z, w)$ on $\widetilde{M}=M \times \mathrm{C}^{n}$, such that condition (4.33) holds. This class, which we will denote by $\varphi^{-1}\left(P^{1}\right)$, is generalized pseudoconvex, i.e. conditions (1.1)-(1.6) hold.

Indeed, (1.1) and (1.5) are obvious (if $g \in C^{(2)} \cap Q$, then so does $g+C$ ). Axiom (1.3) holds, because $\varphi$ is increasing and continuous, and the class $\left(P^{\mathrm{co}}\right)^{d}$, being pseudoconvex, satisfies (1.3) as well. In the same way, (1.4) is checked. As for (1.6), it is enough to observe that the constant function $u_{0}(z, w) \equiv 0$ satisfies condition (4.33). It does, because the function $(z, w) \rightarrow \varphi\left(g(z)+u_{0}(z, w)\right)=\varphi(g(z))$ is constant in $w$, and so is of class $\left(P_{0}^{\text {co }}\right)^{d}$ by Lemma 4.13 .

It is also clear that the class $\varphi^{-1}\left(P^{1}\right)$ is invariant in the sense of axiom (1.9), seeing that both $Q$ and $\left(P^{\text {co }}\right)^{D}$ satisfy (1.9). Thus, the class $\varphi^{-1}\left(P^{1}\right)$ satisfies all the assumptions of Corollary 2.9, and so the class $C_{-\infty, \text { loc }}^{1,1} \cap \varphi^{-1}\left(P^{1}\right)$ is dense in $\varphi^{-1}\left(P^{1}\right)$ in the sense of Corollary 2.9. Since $\varphi$ is $C^{(2)}$-smooth and strictly increasing, we conclude that $C_{-\infty, 10 c}^{1,1} \cap P^{1}$ is dense in $P^{1}$ in the sense of Corollary 2.9, as required in Lemma 3.8.

Part (i) of the theorem is equivalent to the inclusion $P^{1} \subset\left(P^{2}\right)^{d}$. By the above discussion and Lemma 3.8, in order to prove this inclusion, it suffices to show that

$$
Y^{1} \subset\left(Y^{2}\right)^{D}
$$

where $Y^{1}$ and $Y^{2}$ denote the classes of forms representing $P^{1}$ and $P^{2}$, respectively, at $p \in \widetilde{M}$.

Suppose, (4.34) fails, i.e. there is $F \in Y^{1}$, such that $F \notin\left(Y_{Q}^{\text {co }}\right)^{D}$. We fix an admissible coordinate system at $p$ for the rest of the proof and assume that 
form $F(Z \oplus W)$ has representation (4.8), relative to these coordinates. By Lemma 4.12(ii), (iii), form $F$ has the following properties:

$$
\text { the form }\left\langle\bar{W}, A_{22} W\right\rangle+\operatorname{Re}\langle W, B W\rangle \text { is negative definite; }
$$$$
A_{22} \text { is negative definite and }\left(A_{11}-A_{12} A_{22}^{-1} A_{21}\right) \notin Y^{D} \text {. }
$$

(Note that, if the form (4.35) is negative definite, then so is $A_{22}$.) Since the set of all forms satisfying conditions (4.35) and (4.36) is open in $h_{p}$, we can assume without loss of generality (cf. Definition 3.1(c)), that for some function $U=\varphi(u) \in$ $P^{1} \cap C_{-\infty, l o c}^{1,1}$ which has the second-order Peano differential at $p$, it holds

$$
\text { form } F=\operatorname{Hess}^{\mathrm{II}} \varphi \circ u(a) \text { satisfies (4.35) and (4.36). }
$$

Consider now a smooth function $g$ on $M$, such that $g(a)=0, d g(a)=0$. Let $r=u(p)$. By standard computations and (3.2), (3.3), we get

$$
\begin{aligned}
\operatorname{Hess}^{\mathrm{II}} & \varphi(g(\cdot)+u(\cdot, \cdot))(p) \\
= & \varphi^{\prime}(r) \operatorname{Hess}^{\mathrm{C}} g(a)+\varphi^{\prime}(r) \operatorname{Hess}^{\mathrm{II}} u(p) \\
& +\varphi^{\prime \prime}(r) \bar{\partial} u(p) \otimes \partial u(p)+\varphi^{\prime \prime}(r) \operatorname{Re}\left(\partial_{w} u\right)(p) \otimes\left(\partial_{w} u\right)(p),
\end{aligned}
$$

where $\partial$ denotes the first-order complex differential and $\partial_{w}$ its vertical part. Applying this formula with $g \equiv 0$, we obtain

$$
\operatorname{Hess}^{\mathrm{II}} \varphi(g(\cdot)+u(\cdot, \cdot))(p)=\varphi^{\prime}(r) \operatorname{Hess}^{\mathrm{C}} g(a)+\operatorname{Hess}^{\mathrm{II}}(\varphi \circ u)(p),
$$

provided $g(a)=0, d g(a)=0$ and $r=u(p)$.

By (4.36), we can choose $A_{11}^{\prime} \in Y$, such that

$$
\text { the form } A_{11}^{\prime}+\left(A_{11}-A_{12} A_{22}^{-1} A_{21}\right) \text { is negative definite. }
$$

Since the set of the forms $A_{11}^{\prime}$ defined by this condition is open, we can assume without loss of generality, that $A_{11}^{\prime} \in \operatorname{Int}(Y)$, and so, by Lemma 3.11 , there is a smooth function $g_{1}$ of class $Q$, such that Hess ${ }^{\mathbf{C}} g_{1}(a)=A_{11}^{\prime}$. Choose a smooth plurisubharmonic function $u(z)$ near $a$, such that $u(a)=-g_{1}(a)$ and $d u(a)=$ $-d g_{1}(a)$. By axiom (1.8)(i), $\left(u+g_{1}\right) \in Q$, and by assumption (4.7), the function $g(z)=\left(1 / \varphi^{\prime}(r)\right)\left(u+g_{1}\right)(z)$ is of class $Q \cap C^{(2)}$, with $g(a)=0, d g(a)=0$. Hence, by $(4.38), \operatorname{Hess}^{\text {II }} \varphi(g(\cdot)+u(\cdot, \cdot))(p)=A_{11}^{\prime}+F$. Using (4.24), we then obtain

$$
\begin{aligned}
\operatorname{Hess}^{\mathrm{II}} \varphi(g(\cdot)+u(\cdot, \cdot))(p)= & \left\langle\bar{Z},\left(A_{11}^{\prime}+\left(A_{11}-A_{12} A_{22}^{-1} A_{21}\right)\right) Z\right\rangle \\
& -\left|\left(-A_{22}\right)^{-1 / 2} A_{21} Z+\left(-A_{22}\right)^{1 / 2} W\right|+\operatorname{Re}\langle W, B W\rangle .
\end{aligned}
$$

By (4.39), the Hermitian part of this form is negative definite (note, that $A_{22}$ is nonsingular), and the part involving $W$, namely $\left\langle\bar{W}, A_{22} W\right\rangle+\operatorname{Re}\langle W, B W\rangle$, is also negative definite, by assumption (4.35). Lemma 4.13 implies now that the function $\varphi(g(z)+u(z, w))$ cannot be of class $\left(P_{0}^{\text {co }}\right)^{d}$, contrary to the assumption. This proves part (i).

(ii) The proof of this part is very similar to that of (i), with the difference that $P^{2}=P_{Q}^{\mathrm{psh}}$ now, and $P^{1}$ is defined as the set of all usc functions $U(z, w)=$ $\varphi(u,(z, w))$ on $M \times \mathbf{C}^{n}$, such that, for every $g \in C^{(2)} \cap Q$, the function $(z, w) \rightarrow$ $\varphi(g(z)+u(z, w))$ is $(k+n-1)$-plurisubharmonic. We omit further details. 
(iii) This is a partial converse to (i) and (ii) (the actual converse is false) and the proof is very similar to the first part. We will sketch only the $P_{Q}^{\text {co }}$ case; the other case being practically identical.

Consider first an auxiliary class $P$ consisting of all usc functions $u(z, w)$ on $M \times \mathbf{C}^{n}$, such that, for every pluriharmonic function $h(z)$ on $M, \varphi(h(z)+u(z, w)) \in$ $\left(P_{Q}^{\text {co }}\right)^{d}$. Similarly as in part (i), we prove easily that $P$ is an invariant generalized pseudoconvex class, and so the subset $P \cap C_{-\infty, \text { loc }}^{1,1}$ is dense in $P$ in the sense of Corollary 2.9. By this and the fact that $Q \cap C_{-\infty, \text { loc }}^{1,1}$ is also dense in $Q$ in the sense of Corollary 2.9, it suffices to show that $\varphi(g(z)+u(z, w)) \in\left(P_{0}^{\text {co }}\right)^{d}$, when both $g$ and $u$ are of class $C_{-\infty, \text { loc }}^{1,1}$.

Consider any point $x=(z, w)$, such that $u$ and $g$ have the second-order Peano differential at $x$ and $z$, respectively. Choose now a pluriharmonic function $h(z)$ near $x$, such that $h(x)=-g(x)$ and $d h(x)=-d g(x)$. Let $g_{1}=h+g$ and $u_{1}(z, w)=$ $-h(z)+u(z, w)$. Then, $g_{1}(x)=0, d g_{1}(x)=0$, and by (4.38), we get

$$
\operatorname{Hess}^{\mathrm{II}} \varphi(g+u)(x)=\operatorname{Hess}^{\mathrm{II}} \varphi\left(g_{1}+u_{1}\right)(x)=\varphi^{\prime}(r) \operatorname{Hess}^{\mathrm{C}} g_{1}(z)+\operatorname{Hess}^{\mathrm{II}}\left(\varphi \circ u_{1}\right)(x) \text {, }
$$

where $r=u_{1}(x)$. Observe first that

$$
\operatorname{Hess}^{\mathrm{II}} \varphi(g+u)(x) \in\left(Y^{\mathrm{co}}\right)^{D}
$$

(cf. proof of Lemma 4.13). To see this, represent $\varphi^{\prime}(r) \mathrm{Hess}^{\mathrm{C}} g_{1}(z)$ by $\left\langle\bar{Z}, A_{11}^{\prime} Z\right\rangle$, and $\operatorname{Hess}^{\mathrm{II}}\left(\varphi \circ u_{1}\right)(x)$ by (4.8). Then $A_{11}^{\prime} \in Y$ and $\operatorname{Hess}^{\mathrm{II}}\left(\varphi \circ u_{1}\right)(x) \in\left(Y^{\mathrm{co}}\right)^{D}$; cf. Lemma 4.11 for notation. Applying Lemma 4.12(iii) to the latter form, we conclude that either the form $\left\langle\bar{W}, A_{22} W\right\rangle+\operatorname{Re}\langle W, B W\rangle$ is not negative definite, or there is a matrix $S \in \mathbf{C}[n \times k]$, such that

$$
\left(\left(A_{11}^{\prime}+A_{11}\right)+A_{12} S+S^{*} A_{21}+S^{*} A_{22} S\right) \in Y+Y^{D} \text {. }
$$

This relation means that the form

$$
\left\langle\bar{Z},\left(A_{11}^{\prime}+A_{11}\right) Z\right\rangle+\left\langle\bar{Z}, A_{12} W\right\rangle+\left\langle\bar{W}, A_{21} Z\right\rangle+\left\langle\bar{W}, A_{22} W\right\rangle
$$

is not negative definite. Lemma 4.13 implies now that (4.40) holds for a.a. $x$. Eventually, one can use the same argument as in the proof of Lemma 3.8 to prove the next assertion.

A SSERTION. In the situation of Lemma 3.8, if $v \in C_{-\infty, \text { loc }}^{1,1}$ and Hess ${ }^{\mathrm{II}} v(x) \in$ $\left(Y_{x}^{2}\right)^{D}$ for a.a. $x$, then $v \in\left(P^{2}\right)^{d}$. We apply the assertion to $v=\varphi(g+u)$. This completes the proof of part (iii). Q.E.D.

5. Miscellaneous remarks. We will now prove some additional properties of pseudoconvex classes that will be applied in [13].

PROPOSITION 5.1. Let $Q$ be a complex pseudoconvex class on $M=K / H$ (or $P$ be an admissible one on $\widetilde{M}=M \times \mathbf{C}^{n}$ ); cf. (1.1)-(1.9). Assume that

$$
\text { constant functions belong to } Q \text { (or } P \text { ). }
$$

Then $Q^{d d}=Q\left(\right.$ or $\left.P^{d d}=P\right)$.

PROOF. According to [12, Theorem 1.8], $P^{d d}=P$, for an arbitrary pseudoconvex class $P$, provided $\widetilde{M}$ has a basis of $P$-regular neighborhoods in the sense of $[\mathbf{1 2}$, Definition 1.4]. Clearly, if $G$ is a relatively compact neighborhood which is strictly 
convex with respect to an admissible coordinate system, then $G$ is $P$-regular, because, for every $x_{0} \in \partial G$, there exist functions $u_{n}(z, w)$, convex with respect to this coordinate system, such that $u_{n}\left(x_{0}\right)=0, u_{n}(x) \searrow-\infty$, for $x \in \bar{G} \backslash\left\{x_{0}\right\}$. By (5.1) and (1.8), $u_{n} \in P$. Thus, $P^{d d}=P$ (and $Q^{d d}=Q$, as a special case when $n=0)$. Q.E.D.

PROPOSITION 5.2. In the situation of Proposition 3.4, the class of forms representing $P^{d}$ at $p \in \widetilde{M}$ is equal to $Y_{p}^{D}$.

Proof. By Proposition 3.4 and the fact that $Y_{p}^{D}=\mathrm{Cl}\left(\operatorname{Int} Y_{p}^{D}\right.$ ) (cf. [12, Proposition A.5(iv)]), it suffices to find, for a given $F \in \operatorname{Int}\left(Y_{p}^{D}\right)$, a function $u \in C^{2} \cap P$, such that Hess ${ }^{\mathrm{II}} u(p)=F$.

Choose any smooth function, such that $\operatorname{Hess}^{\mathrm{II}} u(p)=F$. In the same way as in the proof of Proposition 3.11(i), we can show that Hess ${ }^{\mathrm{II}} u(x) \in\left(Y_{x}\right)^{D}$ for $x \in V$, where $V$ is a neighborhood of $p$. The assertion at the end of $\S 4$ implies now that $u \in\left(P^{d}\right)(V)$. Q.E.D.

LEMMA 5.3. Let $Q$ be a complex invariant pseudoconvex class on $M=K / H$ satisfying condition (5.1) and let $u \in \operatorname{usc}(U), U \subset M$. Assume that for every holomorphic function $h$ on $M$, the function $\left|e^{h}\right| e^{u} \in Q$. Then $u \in Q(U)$.

PROOF. We use notation and conclusions of Lemma 3.8. Let $P^{1}$ consist of all $u$, such that $\left|e^{h}\right| e^{u} \in Q$, for every holomorphic function $h$. It is easy to check that $P^{1}$ satisfies (1.1)-(1.9). Let $P^{2}=Q^{d}$. Then $P^{2}$ satisfies (1.1)-(1.9) (by [11, Corollary 2.11]). Since $\left(P^{2}\right)^{d}=Q^{d d}=Q$, the lemma is equivalent to the inclusion $P^{1} \subset\left(P^{2}\right)^{d}$. To prove this, it suffices to show, by Lemma 3.8, that $Y_{x}^{1} \subset\left(Y_{x}^{2}\right)^{D}$, $x \in M$. Denote by $Y_{x}$ the representing class of forms for $Q$ at $x \in M$. By Proposition 5.2 and [12, Lemma A.8(iii)], $\left(Y_{x}^{2}\right)^{D}=Y_{x}$. So, we have to prove that Hess $^{\mathbf{C}} u(x) \in Y_{x}$, whenever $u \in P^{1} \cap C_{-\infty, \text { loc }}^{1,1}$. If $h$ is holomorphic, then

$$
\begin{aligned}
\text { Hess }^{\mathrm{C}}\left(\left|e^{h}\right| e^{u}\right)(x)= & \left|e^{h(x)}\right| e^{u(x)} \operatorname{Hess}^{\mathrm{C}} u(x) \\
& +\left|e^{h(x)}\right| e^{u(x)} \overline{\left(\frac{1}{2} \partial h+\partial u\right)} \otimes\left(\frac{1}{2} \partial h+\partial u\right)(x) .
\end{aligned}
$$

By the definition of $P^{1}$, this form belongs to $Y_{x}$; choosing $h$ so that $h(x)=-u(x)$ and $\partial h(x)=-2 \partial u(x)$ ( $x$ fixed), we get Hess ${ }^{\mathrm{C}} u(x) \in Y_{x}$. Q.E.D.

NOTE. Definition 4.1 is closely related to the definition of subharmonic norm functions, introduced independently by Coifman and Semmes in their recent work on interpolation of Banach spaces and nonlinear Dirichlet problems [15]. Both definitions show some similarity to the definition of functions of class $\psi(q)$ given by $\mathrm{Wu}[\mathbf{1 4}]$.

\section{REFERENCES}

1. A. D. Alexandrov, Almost everywhere existence of the second differential of a convex function and properties of convex surfaces connected with it, Leningrad State Univ. Ann. Math. Ser. 6 (1939), 3-35. Russian

2. H. Buseman, Convex surfaces, Interscience, New York, 1958.

3. R. Coifman, M. Cwikel, R. Rochberg, Y. Sagher and G. Weiss, The complex method for interpolation of operators acting on families of Banach spaces, Lecture Notes in Math., Vol. 779, Springer-Verlag, Berlin and New York, 1980, pp. 123-153. 
4. _ $\ldots$ theory of complex interpolation of families of Banach spaces, Adv. in Math. 33 (1982), 203-229.

5. R. L. Hunt and J. J. Murray, q-plurisubharmonic functions and a generalized Dirichlet problem, Michigan Math. J. 25 (1978), 299-316.

6. S. Kobayashi and K. Nomizu, Foundations of differential geometry, Interscience, New York, 1969.

7. S. G. Krantz, Function theory of several complex variables, Wiley, New York, 1982.

8. R. Narasimhan, Analysis on real and complex manifolds, North-Holland, Amsterdam, 1968.

9. Z. Slodkowski, The Bremermann-Dirichlet problem for q-plurisubharmonic functions, Ann. Scuola Norm. Sup. (Pisa) Ser. IV 11 (1984), 303-326.

10. __ Local maximum property and q-plurisubharmonic functions in uniform algebras, J. Math. Anal. Appl. 115 (1986), 105-130.

11. _ Pseudoconvex classes of functions. I, Pseudoconcave and pseudoconvex sets, Pacific J. Math. 134 (1988), 343-376.

12. __ Pseudoconvex classes of functions, II (submitted)

13. __ Complex interpolation of normed and quasi-normed spaces in several dimensions. I, Trans. Amer. Math. Soc. (to appear).

14. H. Wu, On certain Kähler manifolds which are q-complete, Proc. Sympos. Pure Math., vol. 41, Amer. Math. Soc., Providence, R.I., 1984, pp. 253-276.

15. R. Coifman and S. Semmes, Interpolation of Banach spaces and nonlinear Dirichlet problems.

Department of Mathematics, University of Illinois, Chicago, Illinois 60680 